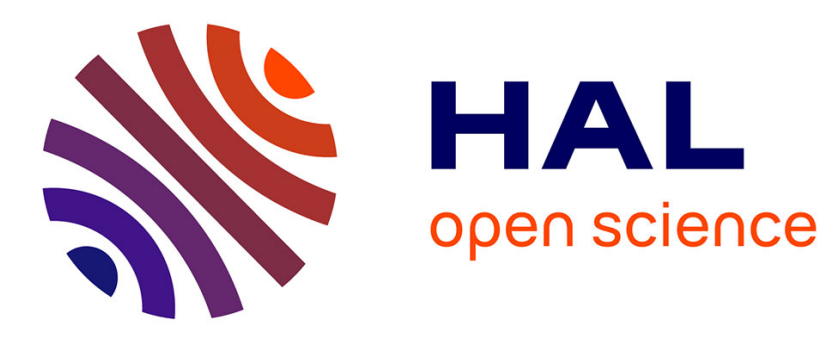

\title{
Three-loop Monte Carlo simulation approach to Multi-State Physics Modeling for system reliability assessment
}

\author{
Wei Wang, Francesco Di Maio, Enrico Zio
}

\section{- To cite this version:}

Wei Wang, Francesco Di Maio, Enrico Zio. Three-loop Monte Carlo simulation approach to MultiState Physics Modeling for system reliability assessment. Reliability Engineering and System Safety, 2017, 167, pp.276-289. 10.1016/j.ress.2017.06.003 . hal-01652211

\section{HAL Id: hal-01652211 \\ https://hal.science/hal-01652211}

Submitted on 30 Nov 2017

HAL is a multi-disciplinary open access archive for the deposit and dissemination of scientific research documents, whether they are published or not. The documents may come from teaching and research institutions in France or abroad, or from public or private research centers.
L'archive ouverte pluridisciplinaire HAL, est destinée au dépôt et à la diffusion de documents scientifiques de niveau recherche, publiés ou non, émanant des établissements d'enseignement et de recherche français ou étrangers, des laboratoires publics ou privés. 


\title{
Three-loop Monte Carlo simulation approach to Multi-State Physics Modeling for system reliability assessment
}

\author{
Wei Wang ${ }^{a}$, Francesco Di Maio ${ }^{\mathrm{a}, *}$, Enrico Zio ${ }^{\mathrm{a}, \mathrm{b}}$ \\ ${ }^{a}$ Energy Department, Politecnico di Milano, Via La Masa 34, Milano 20156, Italy \\ b Chair on System Science and the Energy Challenge, Fondation Electricite' de France (EDF), CentraleSupélec, Université Paris-Saclay, Grande Voie des Vignes, \\ Chatenay-Malabry 92290, France
}

\section{A R T I C L E I N F O}

\section{Keywords:}

Multi-State Physics Modeling

Reliability assessment

Three-loop Monte Carlo simulation

Reactor protection system

Resistance temperature detector

\begin{abstract}
A B S T R A C T
Multi-State Physics Modeling (MSPM) provides a physics-based semi-Markov modeling framework for a more detailed reliability assessment. In this work, a three-loop Monte Carlo (MC) simulation scheme is proposed to operationalize the MSPM approach, quantifying and controlling the uncertainty affecting the system reliability model. The proposed MC simulation scheme involves three steps: (i) the identification of the system components that deserve MSPM, (ii) the quantification of the uncertainties in the MSPM component models and their propagation onto the system-level model, and (iii) the selection of the most suitable modeling alternative that balances the computational demand for the system model solution and the robustness of the system reliability estimates.

A Reactor Protection System (RPS) of a Nuclear Power Plant (NPP) is considered as case study for numerical evaluation.
\end{abstract}

(C) 2017 Elsevier Ltd. All rights reserved.

\section{Introduction}

System reliability assessment relies on a model of the system failure process: the more accurately the model reproduces the system behavior, the more confident the system reliability assessment. Physical knowledge, expert information and data on the system behavior are used to build the model and estimate its parameters [2,3]. The uncertainties in the model and parameters can be propagated by Monte Carlo (MC) simulation [12,47,50,51], Bayesian posterior analysis [46] and Fuzzy methodology [5,18,21,22]. Most commonly, MC simulation is used, consisting in repeatedly sampling random values of the inputs from probability distributions [52].

MSPM is a semi-Markov modeling framework that allows inserting physical knowledge on the system failure process, for improving the system reliability assessment by accounting for the effects of both the stochastic degradation process and the uncertain environmental and operational parameters $[17,30,38,40]$.

In this work, a three-loop MC simulation scheme is proposed for MSPM system reliability modeling. The proposed MC simulation is made of three steps: (i) the identification of the components of the system for which a component-level MSPM is beneficial, because of the importance of the component for the system unreliability, (ii) the quantification and propagation of the uncertainty, and (iii) the selection of the proper mod- eling details, considering computational demand and robustness of the result.

The first step is achieved by Sensitivity Analysis (SA), which can be informed in three different ways: local, regional and global $[16,34]$. Global SA, in particular, measures the output uncertainty over the whole distributions of the input parameters and can be performed by parametric techniques, such as the variance decomposition method $[10,35,36,43,44]$ and moment-independent method [7,8,13,42]. The variance-based method measures the part of the output variance that is attributed to the different inputs or set of inputs, without resorting to any assumption on the form of the model [11,31,33-35]. The momentindependent method allows quantifying the average effect of the input parameters on the reliability of the system and provides their importance ranking [48]. In this work, we resort to moment-independent sensitivity measures, such as Hellinger distance and Kullback-Leibler divergence $[14,20]$, for ranking the input variables most affecting the system reliability uncertainty $[16,24]$.

The second step consists in quantifying the uncertainty in the output of the reliability model. The method adopted for this depends on the components modeling approach: for binary-state Markov Chain Models (MCMs), the variance of the transition failure rate is estimated by Fisher Information Matrix [1,15,26,28]; for MSPM component models, the transition rates uncertainty is propagated and, therefore, estimated by MC.

\footnotetext{
* Corresponding author.

E-mail address: francesco.dimaio@polimi.it (F.D. Maio).
} 


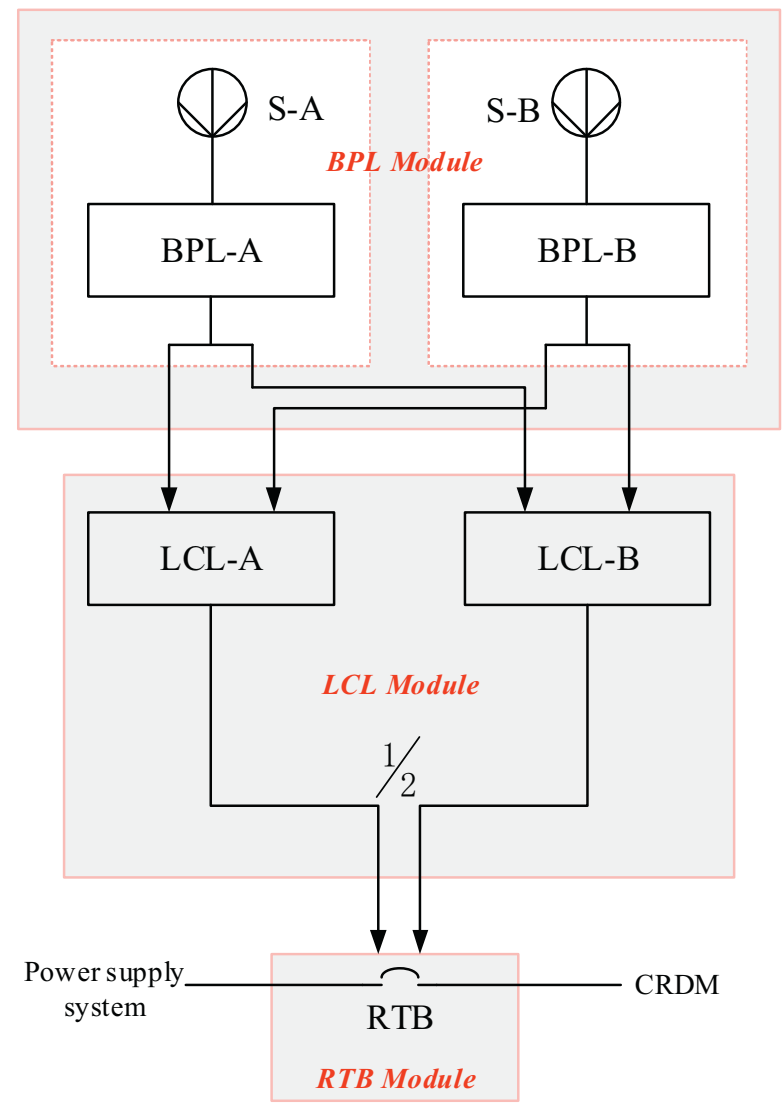

Fig. 1. RPS scheme [41].

For the last step, MC simulation is utilized to propagate uncertainties in the system model and estimate the confidence intervals of the system unreliability.

A Reactor Protection System (RPS) of a Nuclear Power Plant (NPP) is considered as case study. MCM and MSPM are built for the reliability assessment. The Resistance Temperature Detector (RTD) is identified as the most important component. Confidence intervals of the system reliability estimates by RPS-MCM are computed and compared with those of RPS-MSPM that are obtained by the three-loop MC simulation.

The reminder of the paper is organized as follows. Section 2 describes the RPS case study and its MCM reliability model taken as reference. In Section 3, a SA of the MCM is performed and the embedded RTD is identified as the component most affecting the RPS reliability. RPS-MSPM is, then, built for it. Section 4 compares the confidence intervals of the system reliability estimates obtained by MCM and MSPM. In Section 5, conclusions are drawn.

\section{The Reactor Protection System}

The RPS function is to trigger the NPP emergency shutdown, when an anomaly is detected in the measurements of a relevant signal (here assumed to be a temperature signal). As shown in Fig. 1, the RPS is composed of two redundant channels (A and B). Each channel consists of one signal sensor (S-A and S-B), one Bistable Processor Logic (BPL) subsystem (BPL-A and BPL-B), and one Local Coincidence Logic (LCL) subsystem (LCL-A and LCL-B). Usually, redundancy is applied to sensors and signal processing units of RPS. However, with respect to the development of the methods proposed in the paper, we do not consider this for keeping the modeling complexity at a minimum without loss of generality. Furthermore, the sensors S-A and S-B are considered to be RTDs, because of the importance of these components in NPPs digital Instrumentation and Control (I\&C) systems [6,45]. RTDs are safety-

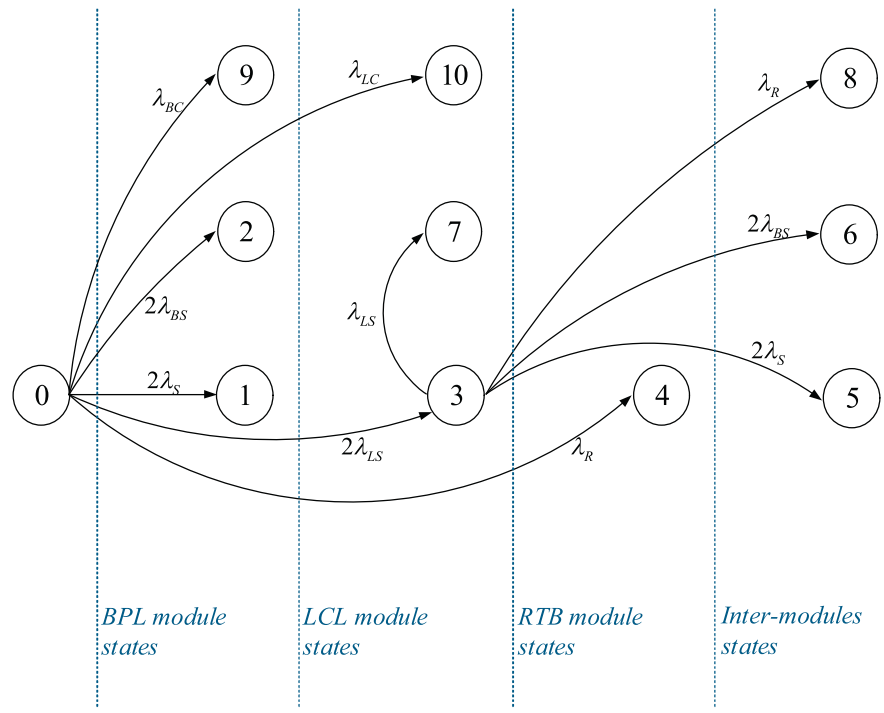

Fig. 2. The RPS-MCM where states are grouped according to their intra-module and intermodules characteristics.

critical components and their effectiveness of detection of anomalous temperatures is very important for plant operators for monitoring the NPP operational conditions [23]. The reliability and accuracy of RTDs is important for controlling the NPP power rate with confidence, guaranteeing large power rates with sufficient safety margins $[40,45]$.

If any one of the two redundant measured signals exceeds a triggering threshold value, a Partial Tripping Signal (PTS) is sent to the corresponding BPL. The signal processing activates only if both channels produce the PTS: each PTS from a BPL is sent to both LCL-A and LCL-B, which process information by an "AND" gate. In other words, an Emergency Shutdown Signal (ESS) is produced only when receiving two PTSs from different BPLs; ESSs, then, activate the Reactor Trip Breaker (RTB), when at least one ESS is triggered, i.e., the information is processed by an "OR" gate. Once the RTB is activated, the power supply system and Control Rod Drive Mechanism (CRDM) which are connected with the RTB activate to control the power of the reactor.

According to the RPS scheme of Fig. 1, three modules are identified:

- The BPL Module consists of two groups of components: sensor and BPL (i.e., "S-A and BPL-A" and "S-B and BPL-B"); these components are connected in series and their failure effects on the system can be combined.

- The LCL Module consists of the two LCLs (i.e., LCL-A and LCL-B); since the ESS is triggered only when both LCLs simultaneously receive two PTSs from the two BPLs, this module is highly dependent of the BPL module.

- The RTB Module.

\subsection{The RPS-MCM}

In this Section, a binary-state MCM is built as reference for the reliability assessment of the RPS. To do this, intra- and inter-module states leading to the system failure are identified. Intra-module states refer to events leading to the system failure that concerns components belonging to the same module; inter-module states relate to system failures from combined component events in different modules.

Fig. 2 shows the RPS-MCM, whose states (listed in Table 1) are grouped into four categories that relate to the intra- and inter-module distinction. The following assumptions have been made for the subsequent quantitative analysis:

- Transitions can occur from the system functioning state (state 0) to any of the absorbing failure states of the intra-module category and 


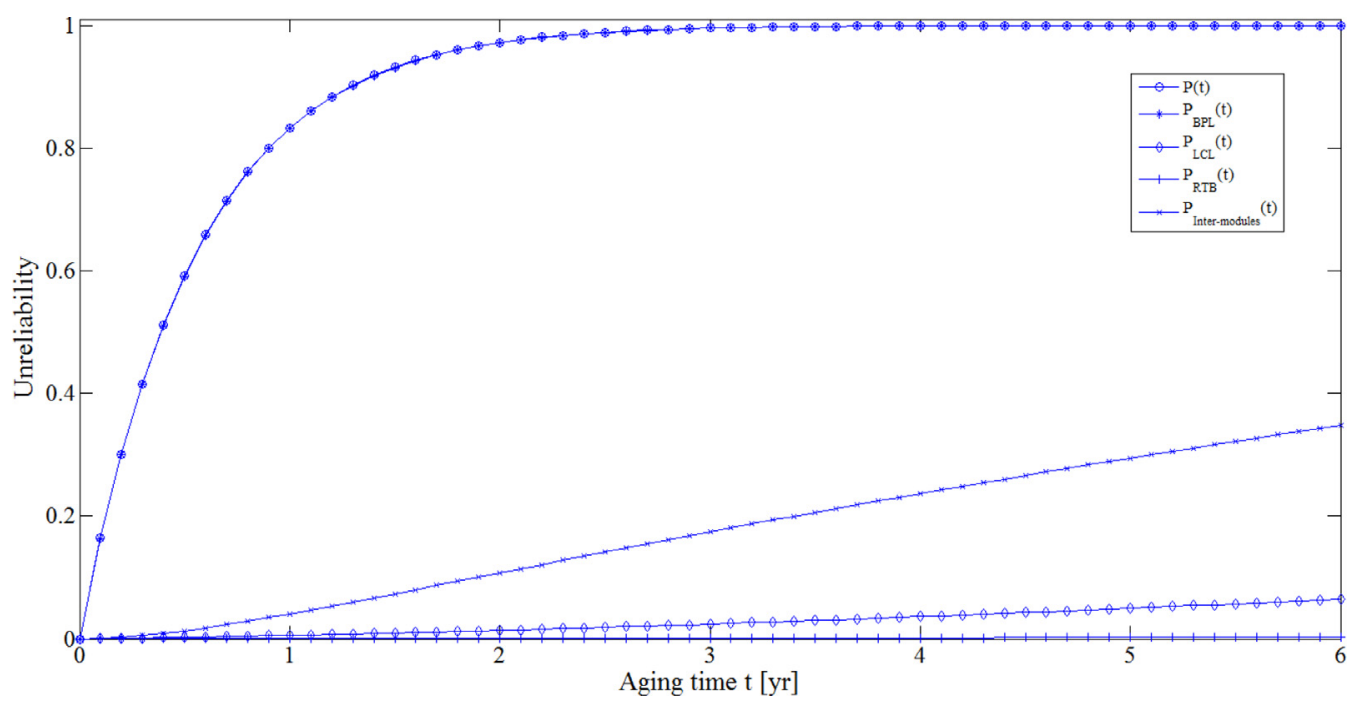

Fig. 3. Unreliability curves of RPS and its modules.

Table 1

Component states.

\begin{tabular}{ll}
\hline State & Description \\
\hline 0 & RPS functioning state. \\
1 & Either one of the RTD sensors fails. \\
2 & Either one of the BPLs fails to send out PTSs. \\
3 & Either one of the LCLs fails to produce the ESS. \\
4 & RTB fails. \\
5 & One LCL has failed and, then, one sensor fails. \\
6 & One LCL has failed and, then, one BPL fails. \\
7 & Both LCLs fail to produce the ESS. \\
8 & One LCL has failed and, then, the RTB fails. \\
9 & Common cause failure of BPL-A and BPL-B. \\
10 & Common cause failure of LCL-A and LCL-B. \\
\hline
\end{tabular}

Table 2

Transition rates [25,39].

\begin{tabular}{lll}
\hline Symbol & Description & Value (/year) \\
\hline$\lambda_{S}$ & RTD failure rate & $8.760 \mathrm{e}-1[39]$ \\
$\lambda_{B}$ & BPL failure rate & $8.760 \mathrm{e}-3[39]$ \\
$\lambda_{L}$ & LCL failure rate & $4.380 \mathrm{e}-2[39]$ \\
$\lambda_{R}$ & RTB failure rate & $3.767 \mathrm{e}-4[25]$ \\
$\beta$ & Common cause factor & 0.1 \\
$\lambda_{B S}$ & BPL self-fault failure rate & $(1-\beta)^{*} \lambda_{B}=7.884 \mathrm{e}-3$ \\
$\lambda_{L S}$ & LCL self-fault failure rate & $(1-\beta)^{*} \lambda_{L}=3.942 \mathrm{e}-2$ \\
$\lambda_{B C}$ & BPLs common cause failure rate & $\beta^{*} \lambda_{B}=8.760 \mathrm{e}-4$ \\
$\lambda_{L C}$ & LCLs common cause failure rate & $\beta^{*} \lambda_{L}=4.380 \mathrm{e}-3$ \\
\hline
\end{tabular}

from the intermediate state (state 3 ) to any of the absorbing states of the inter-module category. The transition rates are taken from public databases $[25,39]$ and reported in Table 2.

- No repairs are considered.

The RPS unreliability $P(t)$, and the individual modules unreliabilities $P_{B P L}(t), P_{L C L}(t), P_{R T B}(t)$ and $P_{\text {Inter-modules }}(t)$ are presented in Fig. 3. A visual analysis of the unreliability curves shows that most of the system unreliability $P(t)$ is contributed by the BPL, that is to say, the absorbing states of the BPL module most contribute to the system unreliability.

\subsection{Uncertainty analysis}

The standard deviation values of the transition rates of Table 2 are either provided by public databases or can be estimated by resorting to Fisher Information $[15,26]$. The procedure for this is here described with reference to the RTD, whose failure rate standard deviation is not provided in [39]:

- Simulation of life tests.

With the mission time $T=6$ years [40] as the end of the rightcensored life tests, we randomly sample $N_{R}=1000$ trials of RTD failure times from an exponential distribution with constant transition rate $\lambda_{S}$ (Table 2). If the sampled time exceeds the mission time $T=6 y e a r s$, the test is considered right-censored [49].

- Estimation of the standard deviation $\hat{\sigma}_{S}$ of $\lambda_{S}$.

The variance of $\lambda_{S}$ can be estimated based on the observed Fisher information [26]. The Fisher Information Matrix is defined from the Maximum Likelihood function or its LogLikelihood [26], and can be estimated by [49]:

$\log L\left(t, \hat{\lambda}_{S}\right)=\log \left(\prod_{i} f_{T}\left(t_{i} ; \hat{\lambda}_{S}\right) \cdot \prod_{j} R\left(t_{j} ; \hat{\lambda}_{S}\right)\right)$

where $i$ and $j$ are the RTD failure times before $T$ and the times rightcensored by $T$, respectively, and $f_{T}\left(t_{i} ; \hat{\lambda}_{S}\right)$ and $R\left(t_{j} ; \hat{\lambda}_{S}\right)$ are the RTD failure time probability density function (pdf) and the RTD reliability:

$f_{T}\left(t_{i} ; \hat{\lambda}_{S}\right)=\hat{\lambda}_{S} \cdot e^{-\hat{\lambda}_{S} t_{i}}$

$R\left(t_{j} ; \hat{\lambda}_{S}\right)=e^{-\hat{\lambda}_{S} t_{i}}$

With respect to the observable random failure time $t$, the Fisher Information Matrix $J\left(\hat{\lambda}_{S}\right)$ can be expressed as:

$J\left(\hat{\lambda}_{S}\right)=E\left[\left(\frac{\partial \log L\left(t ; \hat{\lambda}_{S}\right)}{\partial \hat{\lambda}_{S}}\right)^{2}\right]$

As a result, the variances of the parameters $\hat{\lambda}_{S}$ can be provided from the main diagonal of its inverse matrix $J^{-1}\left(\hat{\lambda}_{S}\right)$, namely, the estimated standard deviations $\hat{\sigma}_{S}$ of the parameters:

$\hat{\sigma}_{S}=J^{-1}\left(\hat{\lambda}_{S}\right)$

Under the condition of mild regularity, $J^{-1}\left(\hat{\lambda}_{S}\right)$ can be calculated by Eq.(6):

$J^{-1}\left(\hat{\lambda}_{S}\right)=\left[-E\left(\frac{\partial^{2} \log L\left(t ; \hat{\lambda}_{S}\right)}{\partial \hat{\lambda}_{S}^{2}}\right)\right]^{-1}$

and the standard deviation can be estimated as: 
Table 3

Estimated transition rates.

\begin{tabular}{lll}
\hline Symbol & Mean value (/year) & Standard deviation (/year) \\
\hline$\lambda_{S}$ & $8.760 \mathrm{e}-1$ & $7.720 \mathrm{e}-1$ \\
$\lambda_{B}$ & $8.760 \mathrm{e}-3$ & $7.867 \mathrm{e}-8$ \\
$\lambda_{L}$ & $4.380 \mathrm{e}-2$ & $1.981 \mathrm{e}-6$ \\
$\lambda_{R}$ & $3.767 \mathrm{e}-4$ & $1.332 \mathrm{e}-10$ \\
\hline
\end{tabular}

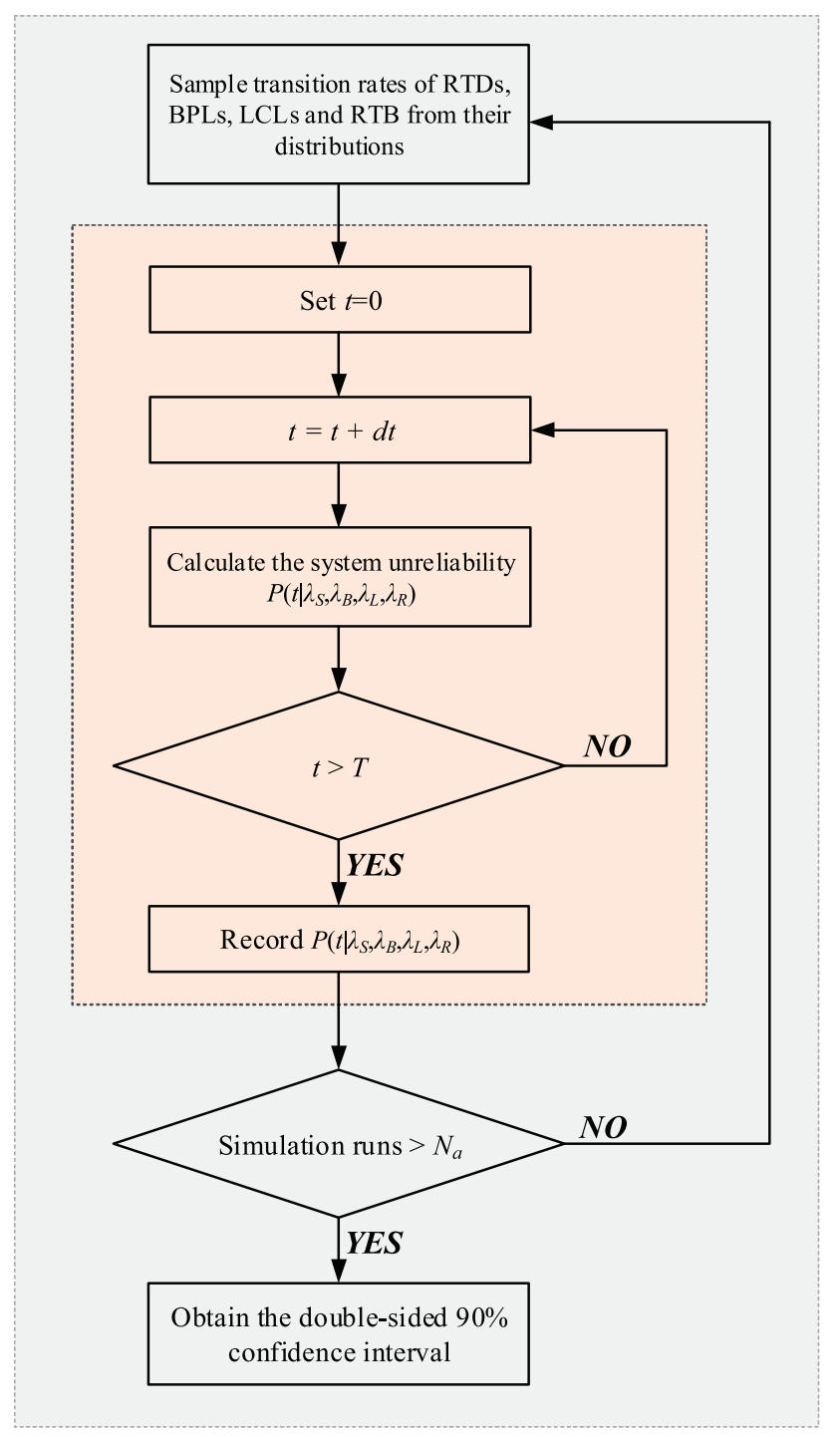

Fig. 4. The flowchart of the two-loop MC simulation for the RPS-MCM system reliability assessment.

$\hat{\sigma}_{S}=J^{-1}\left(\hat{\lambda}_{S}\right)=\left[-E\left(\frac{\partial^{2} \log L\left(t, \hat{\lambda}_{S}\right)}{\partial \hat{\lambda}_{S}^{2}}\right)\right]^{-1}$

The standard deviations of the transition rates of the BPLs, LCLs, and RTB are also estimated by the Fisher Information Methodology (Table 3).

\subsection{Uncertainty propagation}

Uncertainty in binary transition rates is propagated through the RPSMCM as follows (Fig. 4):

(1) Set initial time $t_{0}=0$ and mission time $T=6$ years, and partition the time axis into small intervals of length $d t=0.01$ years;
(2) Sample the component failure rates from the Gaussian distributions $N\left(\lambda_{k}, \hat{\sigma}_{k}\right)$ that are shown in Table 3 , where, $k=S, B, L, R$;

(3) For each time instant $t$ before $T$, compute the system unreliability from the MCM [19,32];

$$
\begin{aligned}
& P\left(t \mid \lambda_{S}, \lambda_{B}, \lambda_{L}, \lambda_{R}\right) \\
& \quad=1-\left(1+\frac{2(1-\beta) \lambda_{L}\left(e^{\left(\beta \lambda_{B}+\lambda_{L}\right) t}-1\right)}{\left(\beta \lambda_{B}+\lambda_{L}\right)}\right) e^{-\left(2 \lambda_{S}+(2-\beta) \lambda_{B}+(2-\beta) \lambda_{L}+\lambda_{R}\right) t}
\end{aligned}
$$

(4) Repeat the steps (2) and (3) for $\mathrm{Na}=1000$ times;

(5) Compute the 5th and 95th percentiles for each time instant $t$.

Fig. 5 shows the plot of the pointwise double-sided 90\% confidence interval of the system unreliability. The confidence interval is large all over the system life $T$, because of the large uncertainty that affects the MCM transition rates due to the weak knowledge utilized to build the, therefore, quite inaccurate RPS-MCM.

\section{RPS-MSPM}

\subsection{The SA approach}

The purpose of this step of the analysis is the identification of the components most important for the system unreliability. This can be a non-trivial problem, for complex systems whose components reliability characteristics (i.e., failure rates) are very uncertain (i.e., with large standard deviations). For clarity, we describe the approach with reference to the case study.

For the RPS components, a MSPM is built for reliability assessment. The SA is performed as follows:

(1) Calculate the moment-independent sensitivity measures between the unreliability $P(t)$ of Fig. 3 and the unreliability $P_{k}(t)$ of its $k$-th module contributor (i.e., $P_{B P L}(t), P_{L C L}(t), P_{R T B}(t)$ and $\left.P_{\text {Inter-modules }}(t)\right)$, to identify the most important module in the system;

(2) Calculate the moment-independent measure for the sensitivity between the module unreliability $P_{k}(t)$ and the unreliability of its $l$-th embedded component $P_{l}(t)$, to identify the component most affecting the module unreliability.

The moment-independent sensitivity measures here adopted are the Hellinger distance and Kullback-Leibler divergence [14,16,20], which rest on the common rationale that the sensitivity measures can be computed as expected generalized distances between the output distribution and the conditional output distribution given the model input(s) of interest [9]. In detail, the Hellinger distance $H_{k}\left[p(t), p_{k}(t)\right]$ measures the difference between the pdf $p(t)$ of the system unreliability and the pdf $p_{k}(t)$ of the $k$-th contributor to the system failure, i.e., BPL, LCL, RTB, Inter-modules [14, 20]:

$$
\begin{aligned}
H_{k}\left[p(t), p_{k}(t)\right] & =\left[\frac{1}{2} \int\left(\sqrt{p(t)}-\sqrt{p_{k}(t)}\right)^{2} d t\right]^{\frac{1}{2}} \\
& =\left[1-\int\left(\sqrt{p(t) \cdot p_{k}(t)}\right)^{2} d t\right]^{\frac{1}{2}}
\end{aligned}
$$

The $k$-th contributor is important if $H_{k}$ is small.

The Kullback-Leibler divergence $K L_{k}\left[p(t), p_{k}(t)\right]$ measures the different information carried by the pdf $p(t)$ of the system failure and the pdf $p_{k}(t)$ of the $k$-th contributor according to Eq. (10) $[14,20]$ :

$K L_{k}\left(p(t), p_{k}(t)\right)=\int_{-\infty}^{+\infty} p(t) \log \left(\frac{p(t)}{p_{k}(t)}\right) d t$

with the values in $[0,+\infty]$. In practical cases, the symmetric form of Kullback-Leibler divergence can be utilized as follows [27]: 


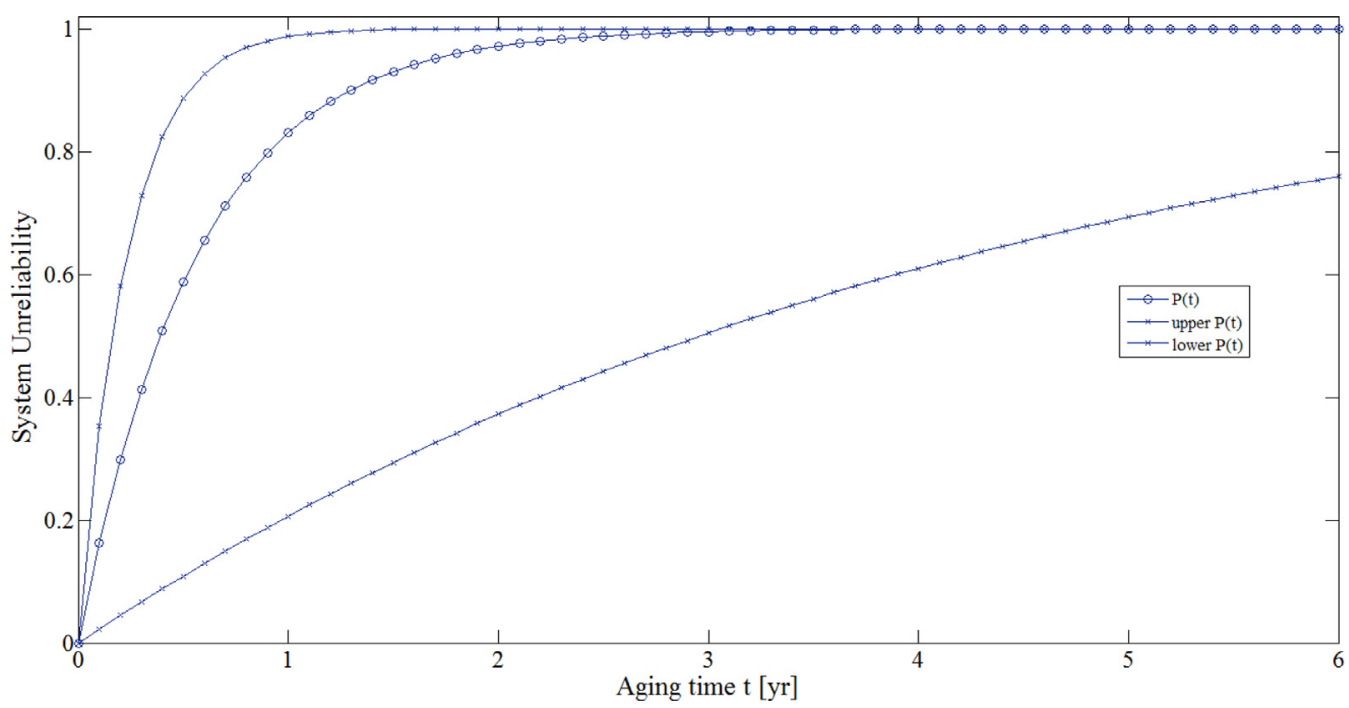

Fig. 5. Confidence intervals from the RPS-MCM system unreliability.

Table 4

Ranking of contributors to the RPS unreliability.

\begin{tabular}{lll}
\hline Input & $\mathrm{H}_{\mathrm{k}}$ & $\mathrm{KL}_{\text {sym, } \mathrm{k}}$ \\
\hline Intra-BPL & 0.0013 & $6.4539 \mathrm{e}-6$ \\
Intra-LCL & 0.6398 & 2.4181 \\
Intra-RTB & 0.6872 & 3.7300 \\
Inter-Module & 0.6000 & 1.8809 \\
\hline
\end{tabular}

Table 5

Ranking of the contributors to the BPL unreliability.

\begin{tabular}{lll}
\hline Input & $\mathrm{H}_{1}$ & $\mathrm{KL}_{\text {sym }, 1}$ \\
\hline Sensors & 0.2391 & 0.2460 \\
BPLs & 0.6219 & 2.1599 \\
\hline
\end{tabular}

$$
\begin{aligned}
K L_{s y m, k}\left[p(t), p_{k}(t)\right] & =K L_{s y m, k}\left[p_{k}(t), p(t)\right] \\
& =\frac{1}{2} K L_{k}\left[p(t), p_{k}(t)\right]+\frac{1}{2} K L_{k}\left[p_{k}(t), p(t)\right]
\end{aligned}
$$

The $k$-th contributor is important if $K L_{s y m, k}$ is small, in relative terms.

\subsubsection{The $S A$ results}

Table 4 lists the Hellinger distance and Kullback-Leibler divergence values for each module contributor to the system unreliability, respectively: both measures identify the BPL as the most important contributor.

Since the BPL module plays the most significant role in affecting the reliability of the RPS, we now focus on identifying the BPL component most contributing to its failure. Fig. 6 shows the unreliability of the BPL module and of the components therein (i.e., $P_{S}(t)$ for the sensor and $P_{B}(t)$ for the BPL-component). The unreliability curves show that most of the BPL module unreliability $P_{B P L}(t)$ is contributed by the sensors, that is to say, the absorbing states of the sensors most contribute to the BPL module unreliability.

To rank the importance of the 1-th component embedded in the BPL module, the two SA measures of Eqs. (9) and (11) are quantified. The sensors turn out to be the most important components contributing to the BPL module unreliability (see Table 5).

\subsection{The RPS-MSPM}

The results of the SA performed in Section 3.1 point at the RTD as the component deserving more modeling efforts for accurate RPS unreliability estimation. A component MSPM is here developed to describe the RTD degradation-to-failure process, inserting physics knowledge in the model.

In general, a MSPM describes the dynamics of component degradation in terms of transitions among a finite number $M$ of degradation states, depending on a parameter vector $\delta$. Similarly to MCM, a state probability $P$ is assigned to each degradation state, forming a state probability vector $P(t, \delta)=\left\{P_{0}(t, \delta), P_{1}(t, \delta), \cdots, P_{j}(t, \delta), \cdots, P_{M}(t, \delta)\right\}$ for all $M$ states [17, 29].

The RTD-MSPM can be integrated into the RPS-MSPM of the RPS, to estimate the system failure probability accounting for both aging- and environmental-dependent transition rates of the RTD.

\subsubsection{The RTD-MSPM}

As discussed in [40], among the RTDs failure modes (e.g., bias, drift, performance degradation, freezing and calibration error), experimental evidence suggests that the main failure mode is drift [4]. Drift is measured by the response time $\tau$ that the RTD needs to reach $63.2 \%$ of a sudden temperature change of the RTD. Aging $t$ and air gap size $\delta$ between the bottom of the thermowell and the sensing tip (that changes because of contamination and mechanical shocks) are the most likely contributors to the drift [23,37]. The response time $\tau(t, \delta)$ is assumed not to exceed the RTD failure threshold $\gamma_{Y}$ during normal operation and in relation to this, the RTD failure boundary is defined as $\partial F=G(t, \delta)=0$, where,

$G(t, \delta)=\tau(t, \delta)-\gamma_{Y}$

The RTD-MSPM shown in Fig. 7 depicts, in a two-state diagram, the partition by $\partial F$ of the safe domain $S$ from the failure domain $F$ of the RTD. The RTD-MSPM assumptions are described as follows:

- $S_{0}^{R T D}$ is the RTD functioning state and $S_{1}^{R T D}$ is the RTD drift failure state;

- Transitions can occur between the two states with failure rate $\lambda_{S}(t \mid \delta)$ and repair rate $\mu_{S}(t \mid \delta)$, functions of the time $t$ and the affecting factor $\delta$;

- At the initial time $t=0$, the RTD is in its initial functioning state $S_{0}^{R T D}$.

3.2.2. Uncertainty analysis of the aging- and environmental-dependent transition rates

To estimate the aging- and environmental-dependent transition rate $\lambda_{S}(t \mid \delta)$, we build the empirical relationship between $\tau$, $t$ and $\delta$ based on experimental data $[23,45]$. In literature, $\tau(t, \delta)$ is usually treated separately, i.e., $\tau(t, 0)$ and $\tau(0, \delta)$. [23] and [45] provide a set of mean response times with standard deviations with respect to the aging time 


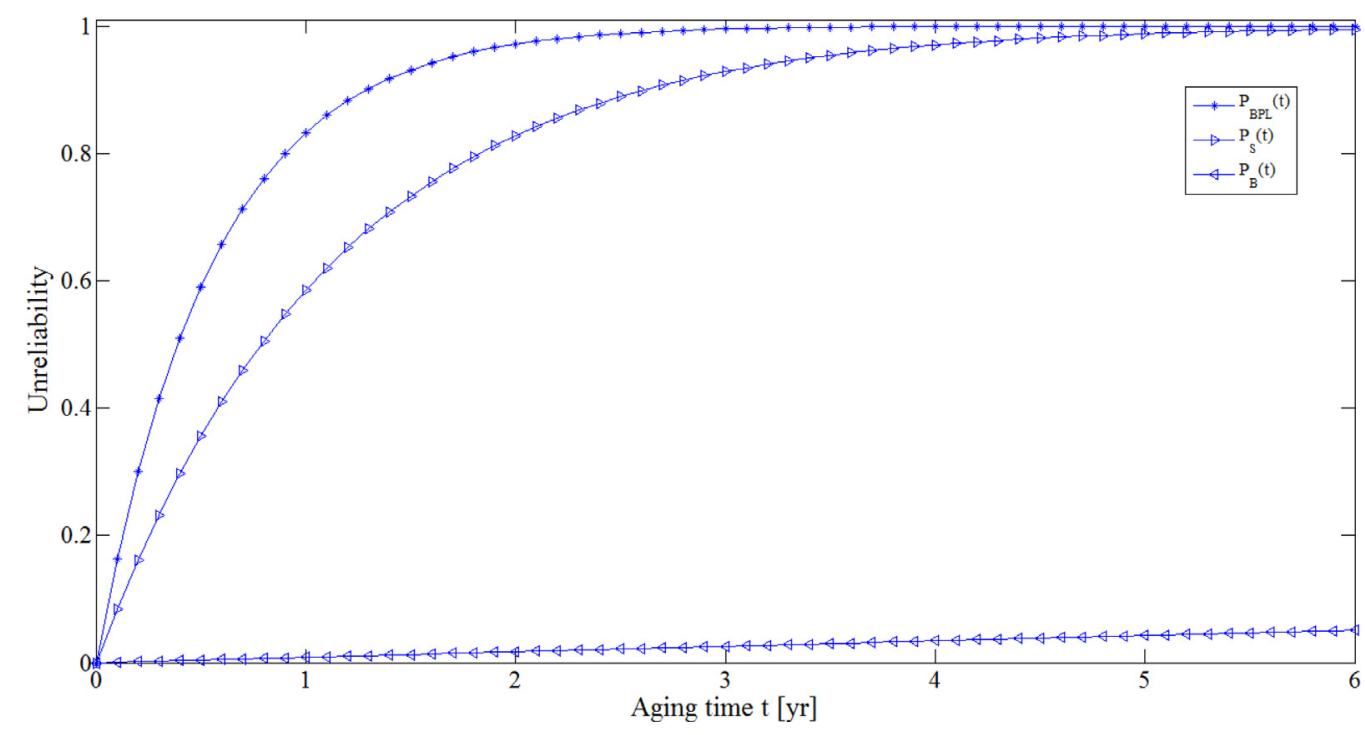

Fig. 6. Unreliability of the BPL module and of its components.

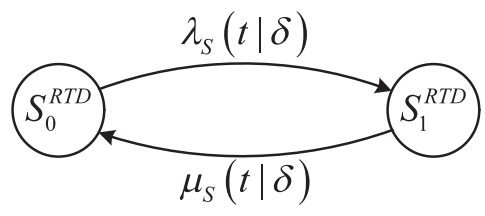

Fig. 7. RTD-MSPM model.

Table 6

Experimental data for $\tau$ at fixed $t$ and $\delta=0$ [45].

\begin{tabular}{llllll}
\hline Aging Time $t$ [year] & 0 & 2 & 4 & 5 & 6 \\
\hline Mean Response Time $\tau$ [s] & 2.1 & 4.4 & 4.8 & 5.0 & 5.2 \\
Standard Deviation $\sigma(t, 0)$ & 1.67 & 0.77 & 0.72 & 0.77 & 0.67 \\
\hline
\end{tabular}

(see Table 6 and Fig. 8, $\delta=0$ ) and a set of mean response times with respect to the air gap size without aging (listed in Table 7, $t=0$ ). Fig. 9 shows the trend of $\tau$ with deviations at discrete $\delta$, when the RTD is new and data in Table 7 are used as interpolation data.

An analytical function of $\tau(t, \delta)$ can be obtained relying on [40]:

$\bar{\tau}(t, \delta)=\alpha_{t} \cdot \bar{\tau}(t-1, \delta)$

$\sigma^{2}(t, \delta)=\sigma^{2}(t-1, \delta)+\alpha_{t}^{2}$

where $\bar{\tau}(t, \delta)$ is the mean value of the response time of Table 7 and Fig. 9, $\sigma(t, \delta)$ is its standard deviation, and the factor $\alpha_{t}$ accounts for the changes of response time $\tau$ with the increase of $t$, by scaling the $\bar{\tau}(t, 0)$ using the scale factor $\alpha_{t}$ :

$\alpha_{t}=\frac{\bar{\tau}(t, 0)}{\bar{\tau}(t-1,0)}$

where, $\alpha_{1}=\bar{\tau}(1,0) / \bar{\tau}(0,0)$.

The function $\tau(t, \delta)$ consists in a surface fitted to realizations of $\tau(t, \delta)$ sampled from the assumed Gaussian distributions with mean values $\bar{\tau}(t, \delta)$ and standard deviations $\sigma(t, \delta)$ at each discrete point, as shown in Fig. 10 where one trial surface is plotted.

The transition rate $\lambda_{S}(t \mid \delta)$ and the cumulative distribution function (cdf) $P_{S}(t \mid \delta)$ can, thus, be estimated as the probability of $\tau(t, \delta)$ to exceed $\gamma_{Y}=8 \mathrm{~s}$ at a given time $t$, based on a batch of MC simulations that are run as described in detail in [40]. For the sake of clarity, the estimated $\lambda_{S}(t \mid \delta)$ for the surface of Fig. 10, obtained by simulating $N b=1000$ different degradation processes, is plotted in Fig. 11.
It is worth mentioning that each trial surface results in a different expression of $\tau(t, \delta)$, RTD failure boundary $\partial F$, transition rate $\lambda_{S}(t \mid \delta)$ and the cdf, due to the randomly sampled discrete response times upon which the surface is built on.

Therefore, the accuracy of the RTD-MSPM, to a large extent, depends on the uncertainties of the RTD physical parameters that are used to build the surfaces. Indeed, the variance of the sampled $\tau(t, \delta)$ greatly affects the output of the MSPM model, the RTD degradation process and the probability of failure boundary $\partial F$ exceedance for each time.

\subsubsection{Three-loop MC simulation for uncertainty propagation through the RPS-MSPM}

The RPS-MSPM model of Fig. 12 embeds the RTD-MSPM model of Fig. 7, while components other than the RTD are assumed to obey binary-state behaviors as in the reference MCM of Fig. 2.

We propose the three-loop MC simulation for the RPS reliability assessment, with confidence quantification related to the uncertainty in the RTD physical parameters propagated through the surfaces $\tau(t, \delta)$ of Section 3.2.2, and in the transition rates for the binary components that are accounted for by the Fisher Information Matrix of Section 2.2. The outmost loop within the following procedure (sketched also in Fig. 13) consists in randomly sampling the values of the physical RPS model parameters from their distributions and sampling the RTD failure time (step (4)):

(1) Set initial time $t_{0}=0$, mission time $T=6$ years and time step $d t=0.01$ years;

(2) Randomly sample the transition rates of the binary-states components (i.e., BPLs, LCLs, and RTB) from the Gaussian distributions $N\left(\lambda_{k}, \hat{\sigma}_{k}\right)$ of Table 3 , where, $k=\mathrm{B}, \mathrm{L}, \mathrm{R}$;

(3) Sample the failure times of the binary-states components, from the exponential distributions with the sampled transition rates;

(4) Randomly sample the multi-state RTD failure time by:

(4a) Fit the randomly sampled realizations of the RTD response time $\tau$ at each discrete point to a trial surface $\tau(t, \delta)$;

(4b) Simulate the RTD degradation process evolution from $t=t_{0}$ to $t=T$;

(4c) At each time $t$, sample the air gap size increment $d \delta_{t}$ from a normal distribution $N(0,0.025 t)$, resulting in $\delta=\delta_{0}+\mathrm{d} \delta_{t}$.

(4d) Calculate the response time $\tau$ on the fitted trial surface $\tau(t, \delta)$.

(4e) Record the time $t$ at which $\tau$ exceeds the threshold $\gamma_{Y}=8 \mathrm{~s}$, with air gap size $\delta$.

(5) Integrate the RTD-MSPM into the RPS-MSPM; 


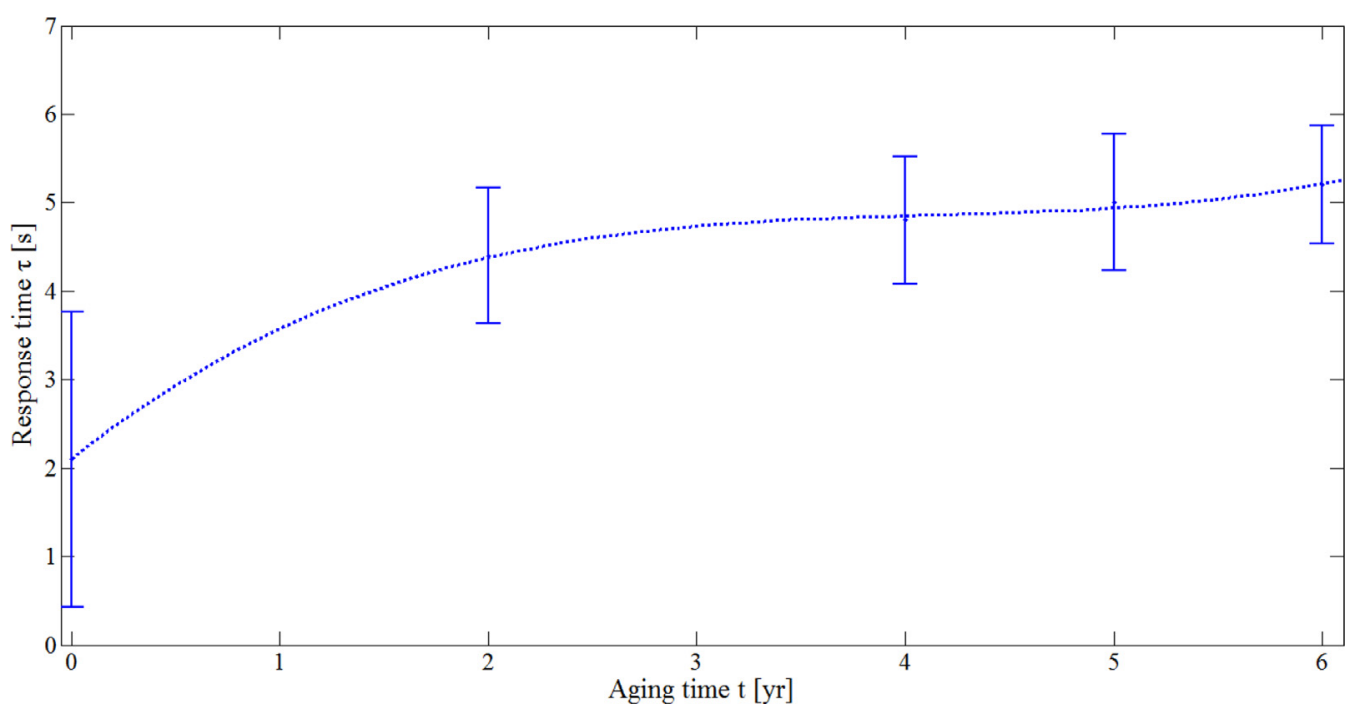

Fig. 8. $\tau(t, 0)$ with standard deviations.

Table 7

Fitted $\tau$ at $t=0$ and discrete $\delta$ based on experimental data from [23,45].

\begin{tabular}{llllllll}
\hline Air gap size $\delta[\mathrm{mm}]$ & 0 & 0.4 & 0.8 & 1.2 & 1.6 & 2.0 & 2.4 \\
\hline Mean response time $t[\mathrm{~s}]$ & 2.10 & 3.80 & 4.97 & 5.93 & 7.02 & 8.58 & 10.95 \\
Standard deviation $\sigma(0, \delta)$ & 1.18 & 1.19 & 1.64 & 2.47 & 3.61 & 4.98 & 6.51
\end{tabular}

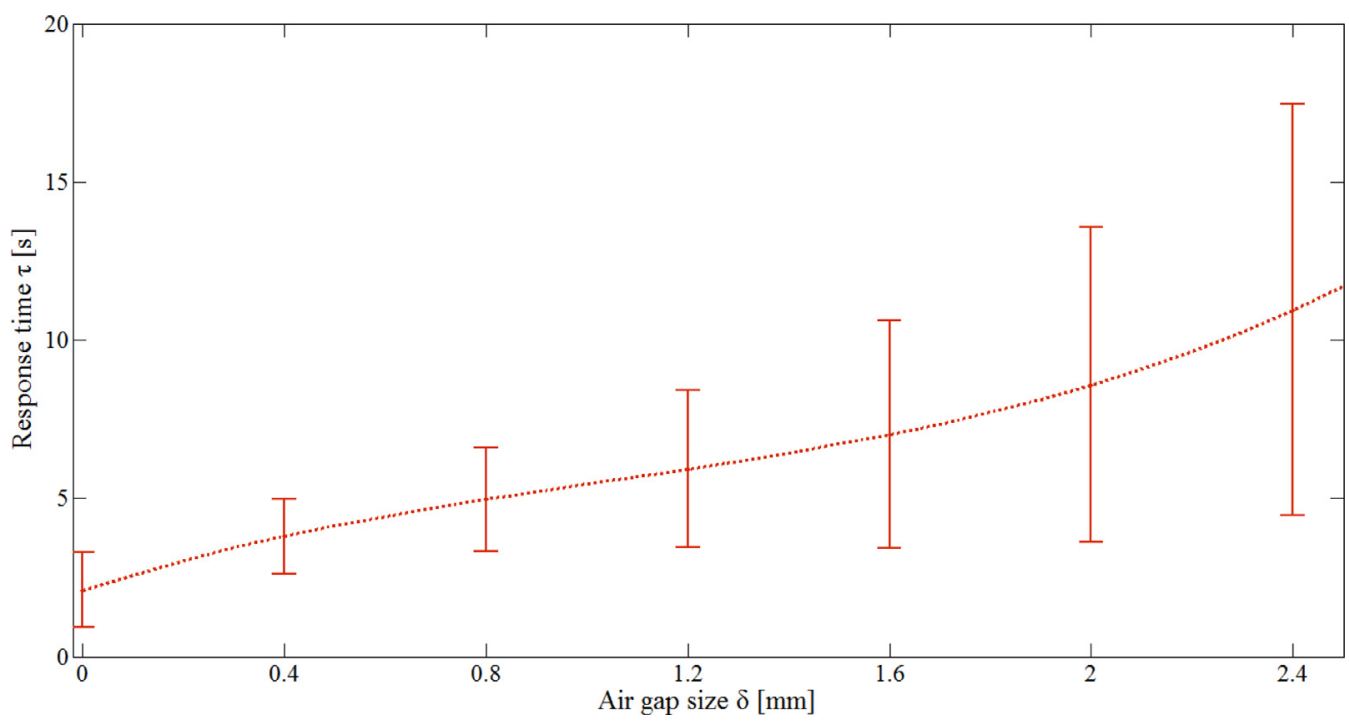

Fig. 9. $\tau(0, \delta)$ with standard deviations.

(5a) Sort all the components sampled failure times;

(5b) Check whether the minimum of the sorted times exceeds $T$ :

- If yes, increase the unreliability counter at time $T$;

- If not, check whether at that time the RPS-MSPM reaches any absorbing state and, if yes, increase the unreliability counter, or the reliability counter, otherwise.

(6) Run $N b=1000$ times steps (1) to (5) to build the empirical $P\left(t \mid \delta ; \lambda_{B} ; \lambda_{L} ; \lambda_{R}\right)$, based on the statistics of the system unreliability estimates collected at each time $t$;

(7) Estimate the 5th and 95th percentiles of the unreliability by repeating steps (1) to (6) for $N c=1000$ times and collecting the related statistics;

(8) Obtain the pointwise double-sided $90 \%$ confidence intervals of the system unreliability calculated by the RPS-MSPM.
Fig. 14 shows the estimated $\mathrm{P}(t, \delta)$ with the $90 \%$ confidence interval for the RPS-MSPM of Fig. 12, obtained by the three-loop MC simulation. The confidence interval is large especially in $[0.5,1.5]$ years, probably because the fitted trial surfaces at the basis of the uncertainty propagation considerably vary from each other due to the large variances of the data of Tables 6 and 7 utilized to build them. Despite that, as we shall see in what follows, the robustness of the assessment is much improved with respect to the RPS-MCM results.

\section{Comparison}

Fig. 15 shows the results of the RPS reliability assessment by the RPSMCM of Section 2 and the RPS-MSPM of Section 3. In general terms, it can be concluded that the RPS-MSPM results provide a narrower confidence interval than the RPS-MCM, thanks to the integration of physics 


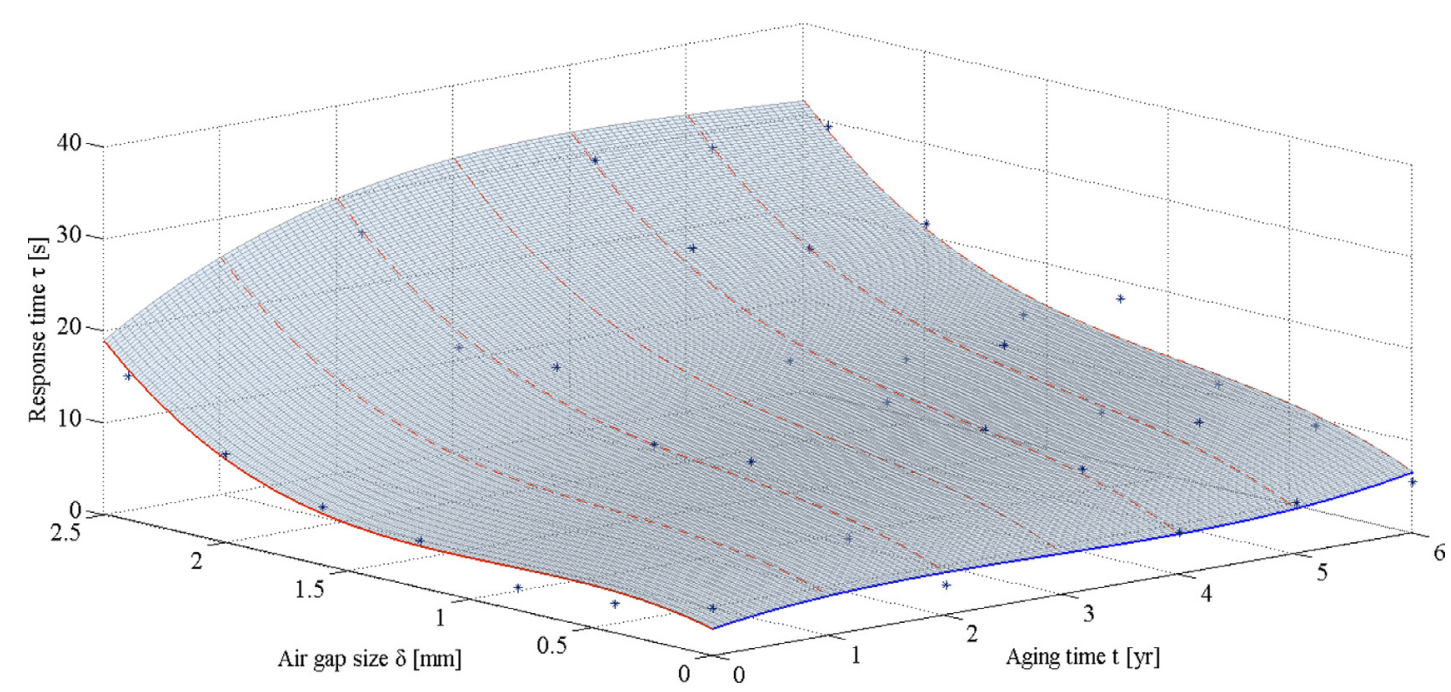

Fig. 10. Fitted surface of $\tau(t, \delta)$.

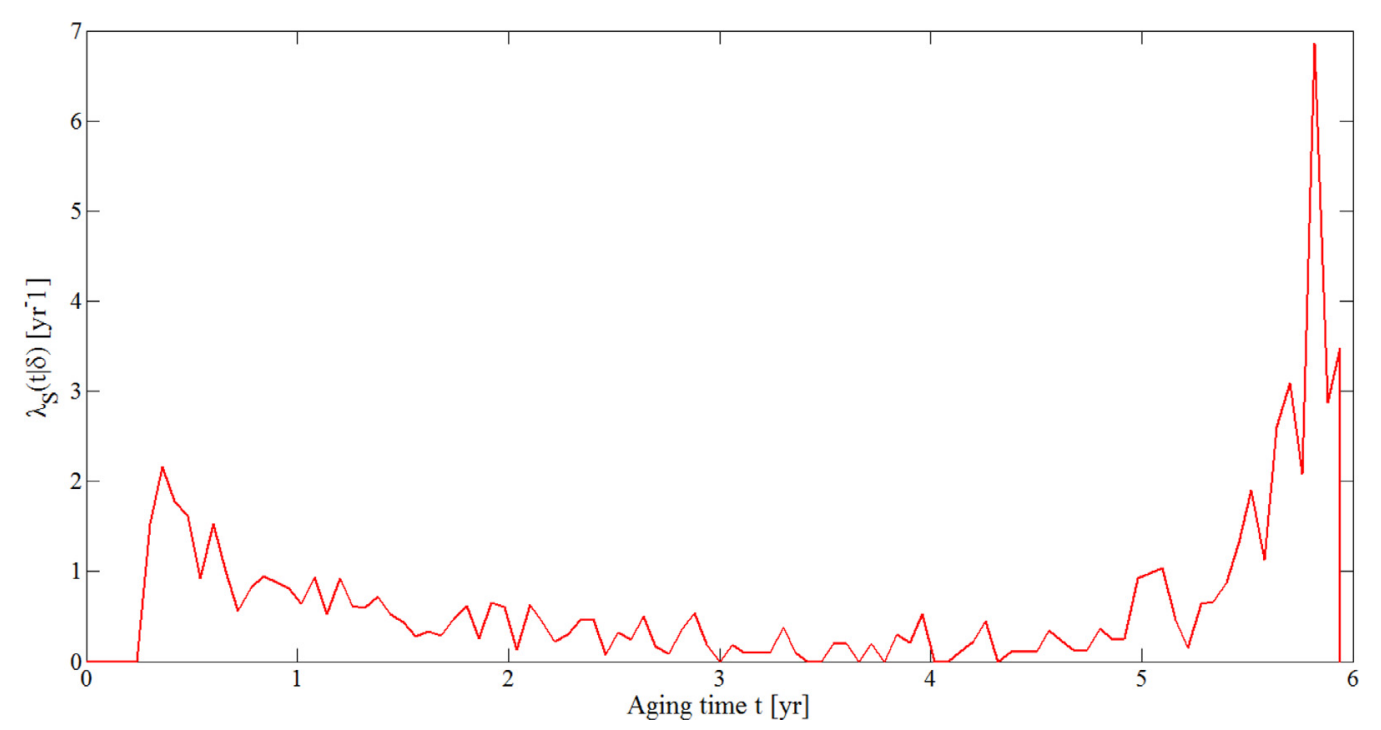

Fig. 11. Conditional failure rate $\lambda_{S}(t \mid \delta)$ of RTD new-to-drift failure mode.

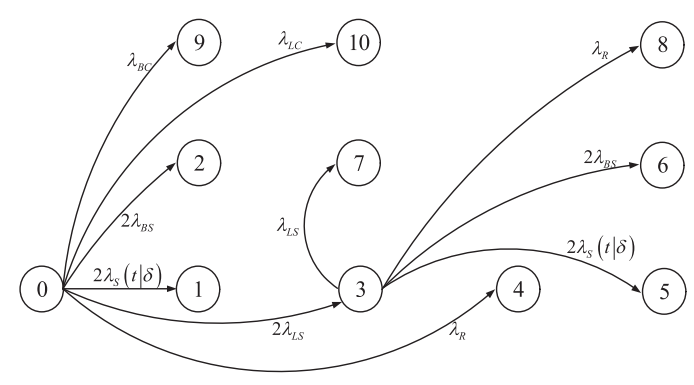

Fig. 12. RPS-MSPM integrating the RTD-MSPM.

knowledge related to operational and environmental parameters. The confidence interval provided by the MSPM is larger than that of the MCM at the early stage of the RPS life ( $t<1$ year): the main reason is that the fitting surfaces may considerably vary from trial to trial due to the large variance of the response times at the considered discrete points, which greatly affect the onset time of the RTD drift failure mode.

For a quantification analysis, two indexes (i.e., the relative uncertainty interval width $\zeta_{t}$ and the relative age interval width $\zeta_{P}$ ) are pro- posed in what follows to compare the accuracy of the MCM with that of the MSPM.

\subsection{The relative unreliability interval width}

At each time $t$, the ratio $\zeta_{t}$ between the mean value of the system unreliability and the width of the unreliability interval (i.e., the difference between the upper and lower bounds) is calculated.

The larger $\zeta_{t}$, the narrower is the confidence interval, and the more accurate the system reliability modeling approach. Fig. 16 shows that $\zeta_{t}(t \mid \delta)$ of the MSPM is much larger than $\zeta_{t}(t)$ of the MCM: as $t$ increases, the estimated system unreliability obviously increases but, since MSPM includes more (physics) knowledge on the system behavior than MCM, the confidence interval reduces more than that of the MCM. The zoom of Fig. 16 shows the evolution of $\zeta_{t}$ from $t=0$ to $t=2$ years: to further investigate the dispersion of the unreliability estimates within the bounds, we calculate, at each time, their empirical pdf and the respective cdf.

Ideally, we would prefer a reliability assessment method that provides distributions peaked on the mean value (rather than uniform distributions), because this would facilitate the decision maker that would, then, be more prone to accept the mean reliability value. Fig. 17 shows an example of possible empirical distributions (at time $t=1$ year, with- 


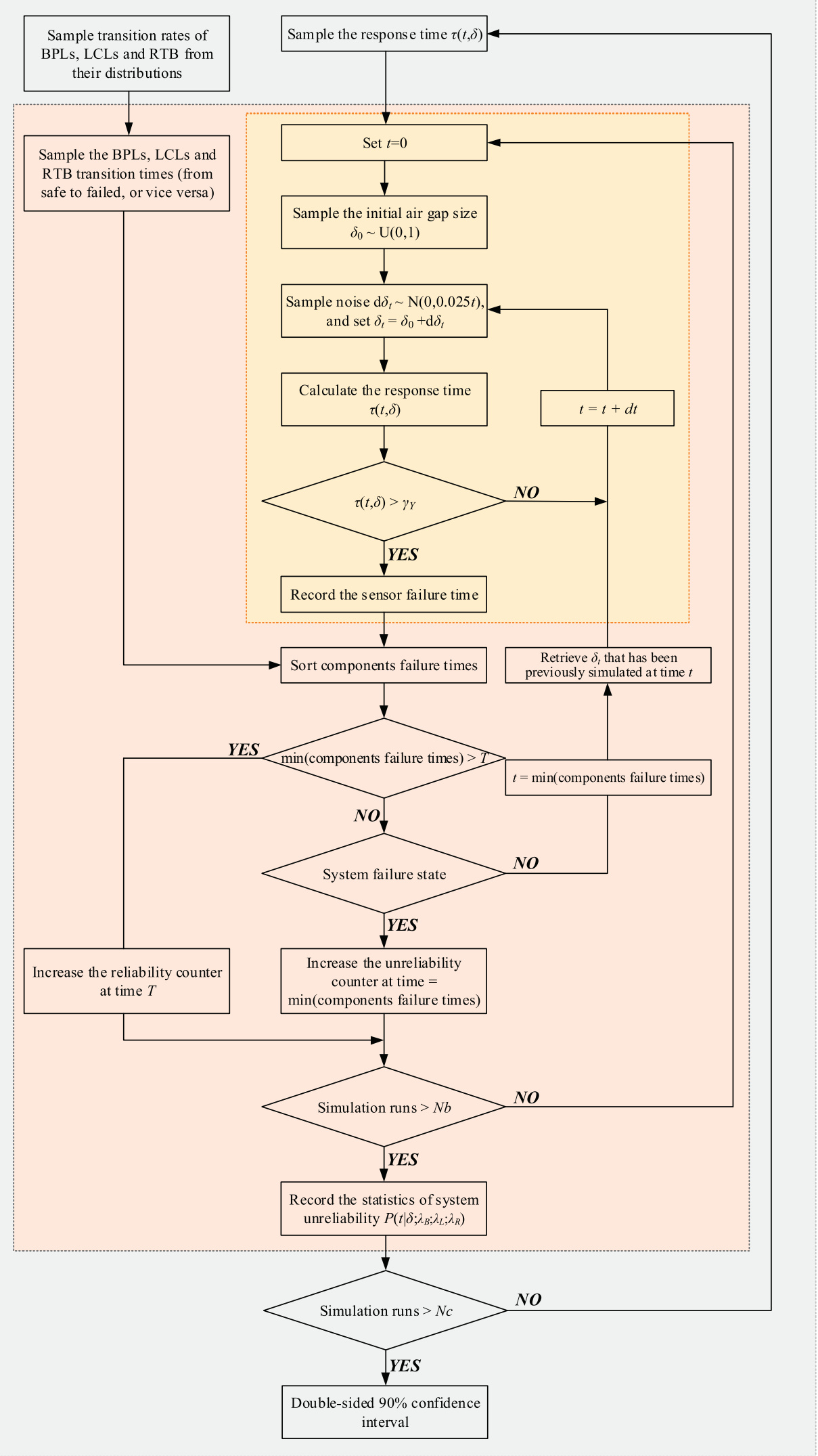

Fig. 13. Flowchart of the three-loop MC simulation. 


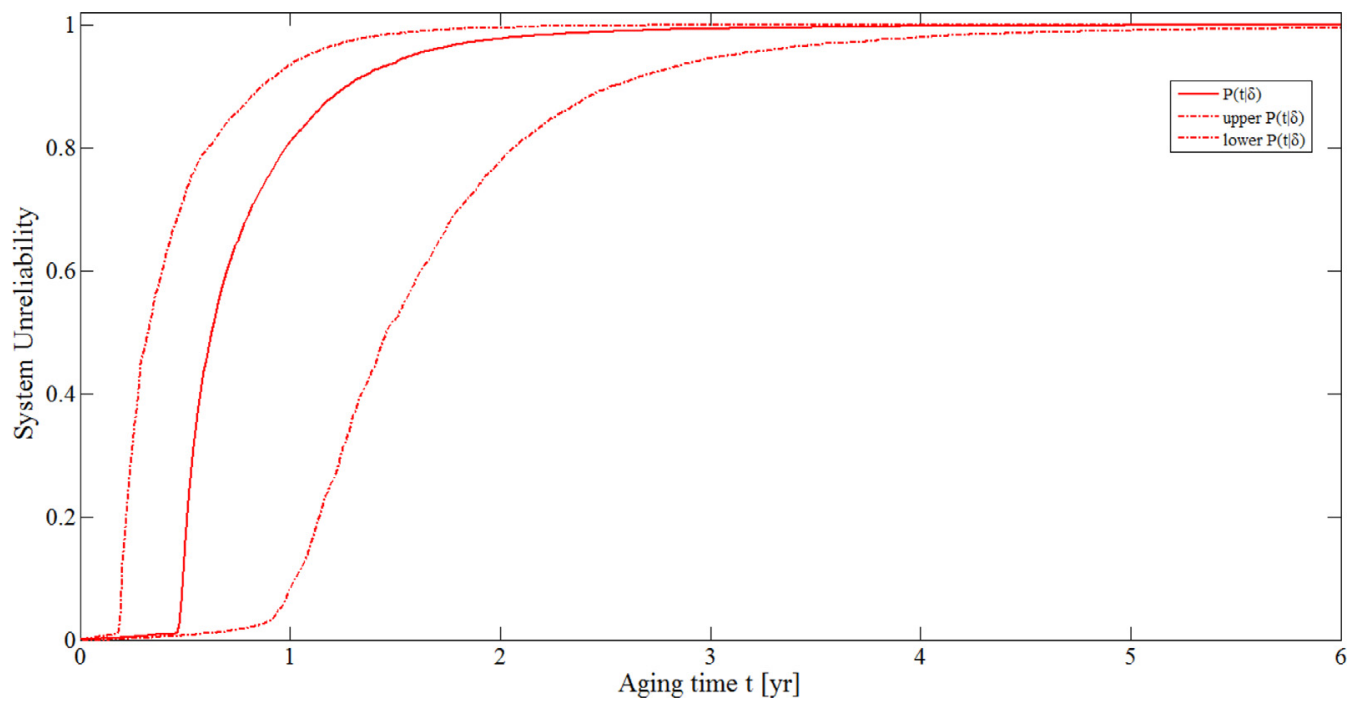

Fig. 14. Estimated RPS-MSPM unreliability with $90 \%$ confidence interval.

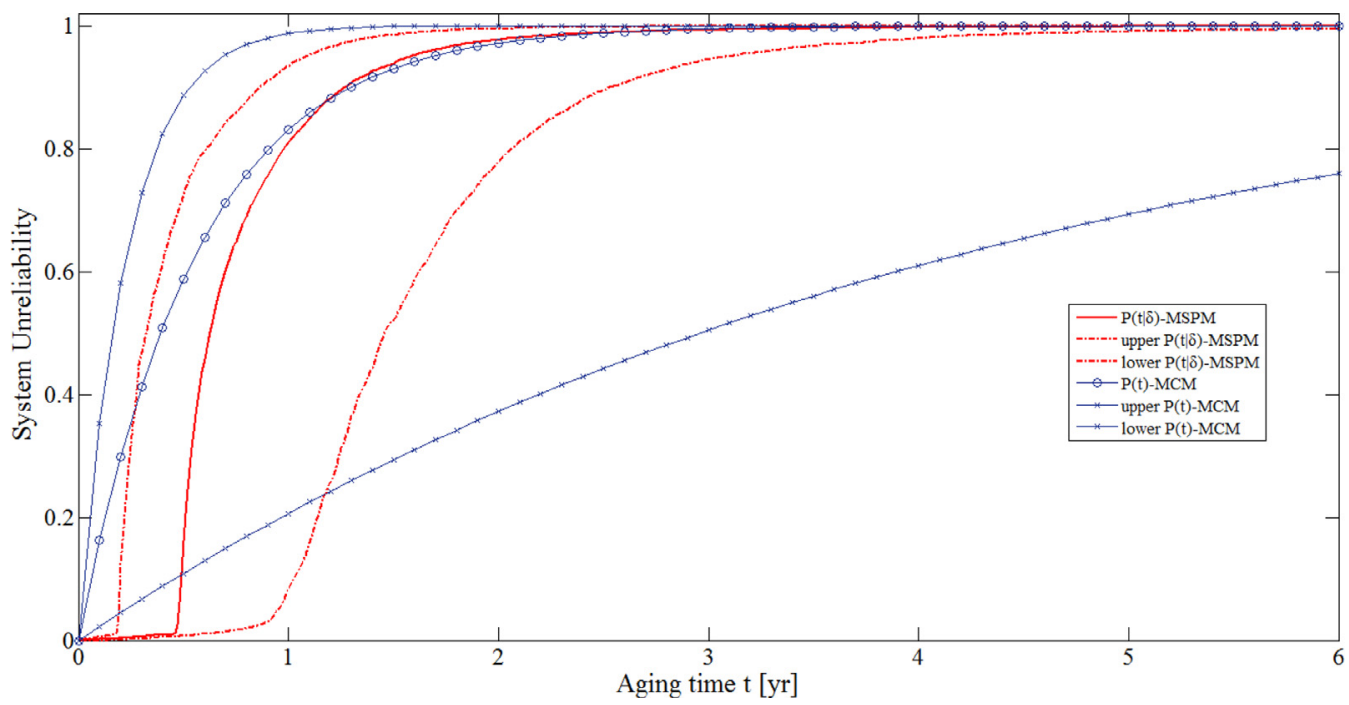

Fig. 15. System unreliability with confidence intervals, provided by MCM and MSPM.

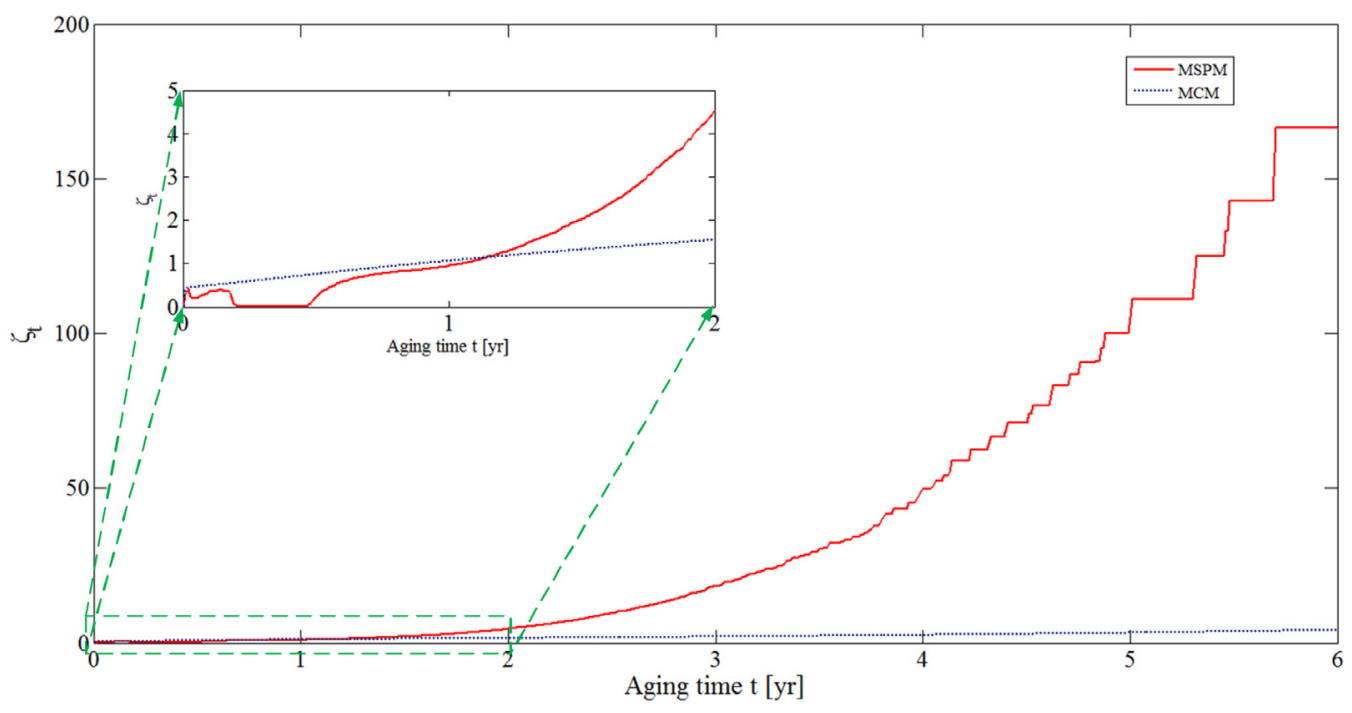

Fig. 16. Relative unreliability interval width. 


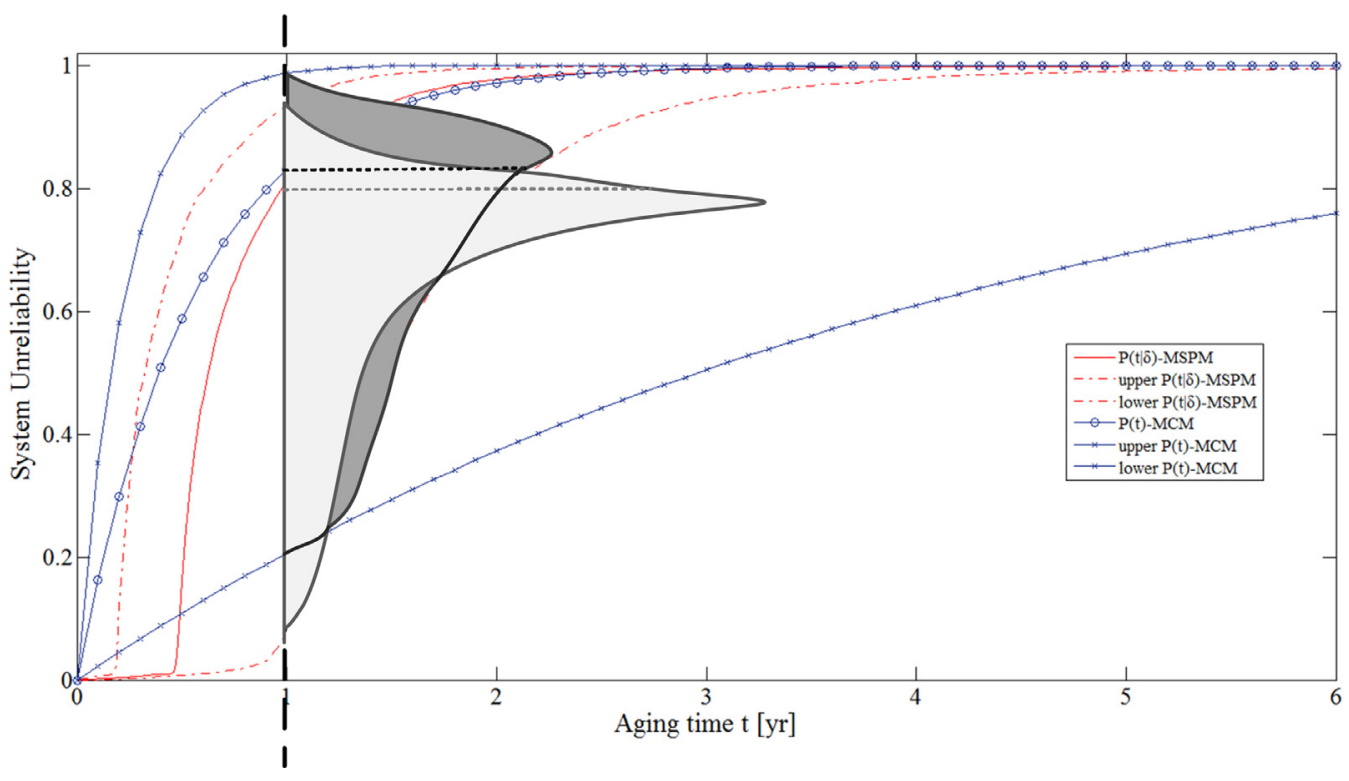

Fig. 17. Example of empirical distributions of the unreliability value at $t=1$ year.

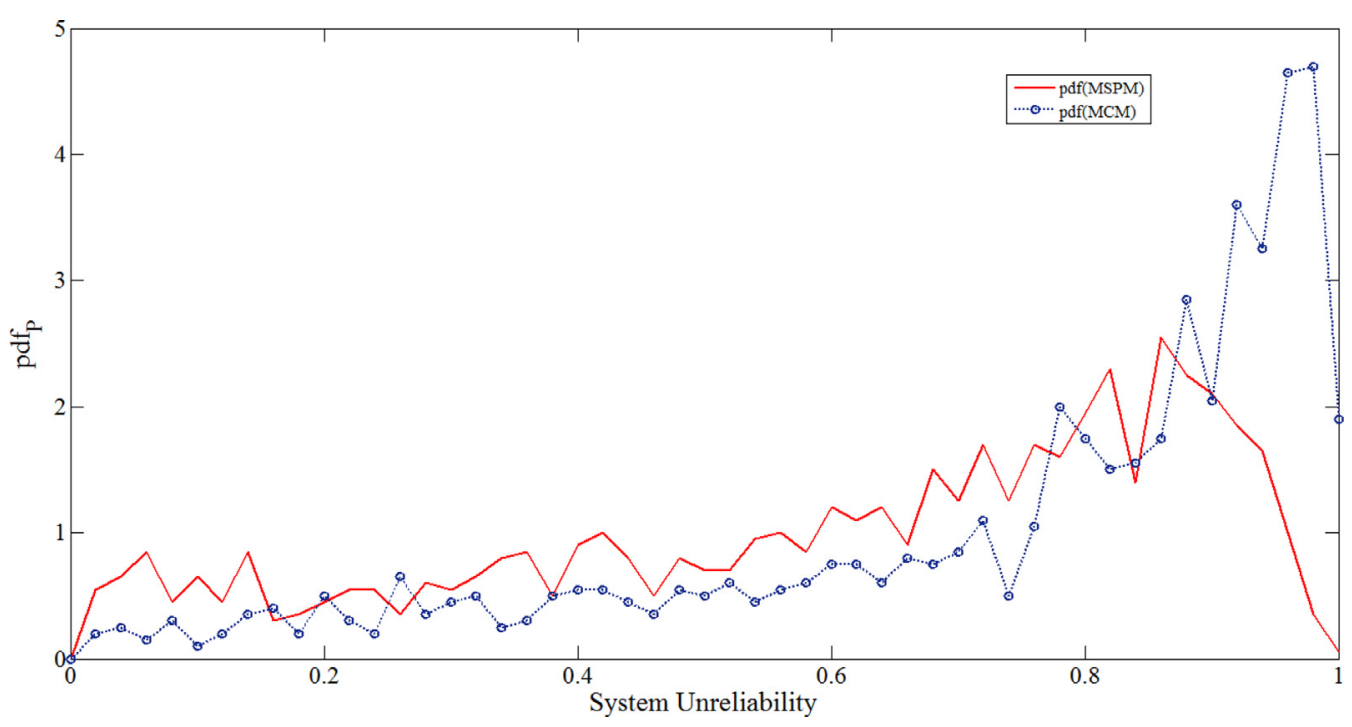

Fig. 18. pdf of the MCM estimates vs. pdf of the MSPM estimates at $t=1$ year.

out any loss of generality). The decision maker would rely on the MSPM rather than the MCM (light and dark shadowed areas, respectively), because in the latter case most of the unreliability estimates confirm the MSPM to be more accurate than the MCM.

Based on the real estimates collected with the $\mathrm{Na}$ MC simulations for the RPS-MCM reliability assessment of Section 2 and the Nc three-loop MC simulation for the RPS-MSPM of Section 3, Figs. 18 and 19 show the $\mathrm{pdf}$ and cdf curves of the system unreliability at $t=1$ year. The pdf of the MCM, as well as its cdf, skews towards large unreliability values, compared to the pdf of the MSPM, demonstrating again the more probable overestimation of the system unreliability, if the decision maker were to resort to RPS-MCM.

\subsection{The relative age interval width}

With respect to each system reliability value $P$, the ratio $\zeta_{P}$ between the mean value of the system failure time and the width of the age interval (i.e., the difference between the upper and lower bounds), is calculated.
The larger $\zeta_{P}$, the narrower the confidence interval, and the more accurate the system reliability estimate. Fig. 20 shows $\zeta_{P}(P \mid \delta)$ of the MSPM and $\zeta_{P}(P)$ of the MCM. The latter is always larger than the former, whatever the value of $P$, that means that MSPM better models the RTD degradation and, therefore, provides more accurate failure time predictions than the MCM. For clarity sake, $\zeta_{P}(P)$ of the MCM is truncated at $P=0.8$ because the maximum unreliability of the lower bound of the MCM is 0.8 within the mission time.

To further investigate the dispersion of the age interval estimates, we calculate, at each unreliability value $P$, the empirical pdf and respective cdf. Ideally, we would prefer a reliability assessment method that provides distributions peaked on the mean value of the failure time. Fig. 21 shows an example of possible empirical distributions (at $P=0.1$, without any loss of generality). The decision-maker would rely on the MSPM rather than the MCM (light and dark shadowed areas, respectively) because in the latter case most of the failure time estimates confirm the MSPM to be more accurate than the MCM.

Resorting to the real estimates collected with the $\mathrm{Na}$ MC simulation for the RPS-MCM reliability assessment of Section 2 and the Nc three- 


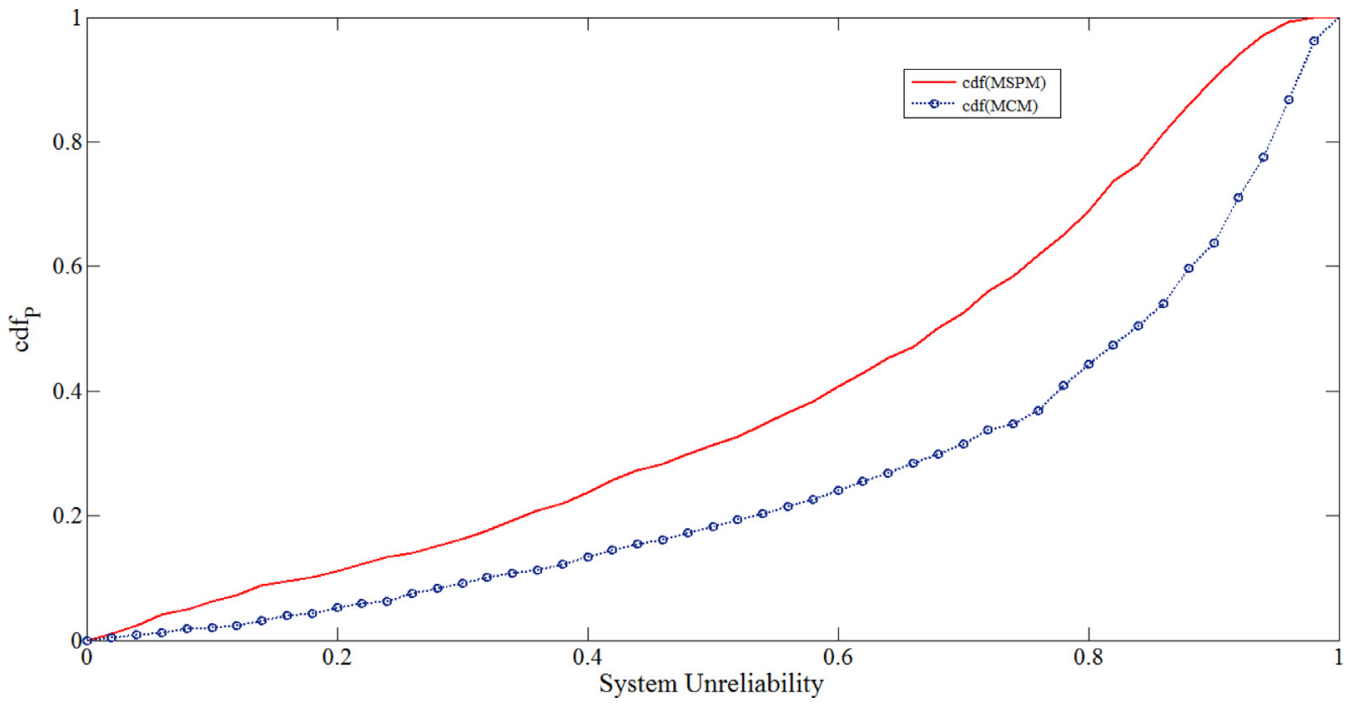

Fig. 19. cdf of the MCM estimates vs. cdf of the MSPM estimates at $t=1$ year .

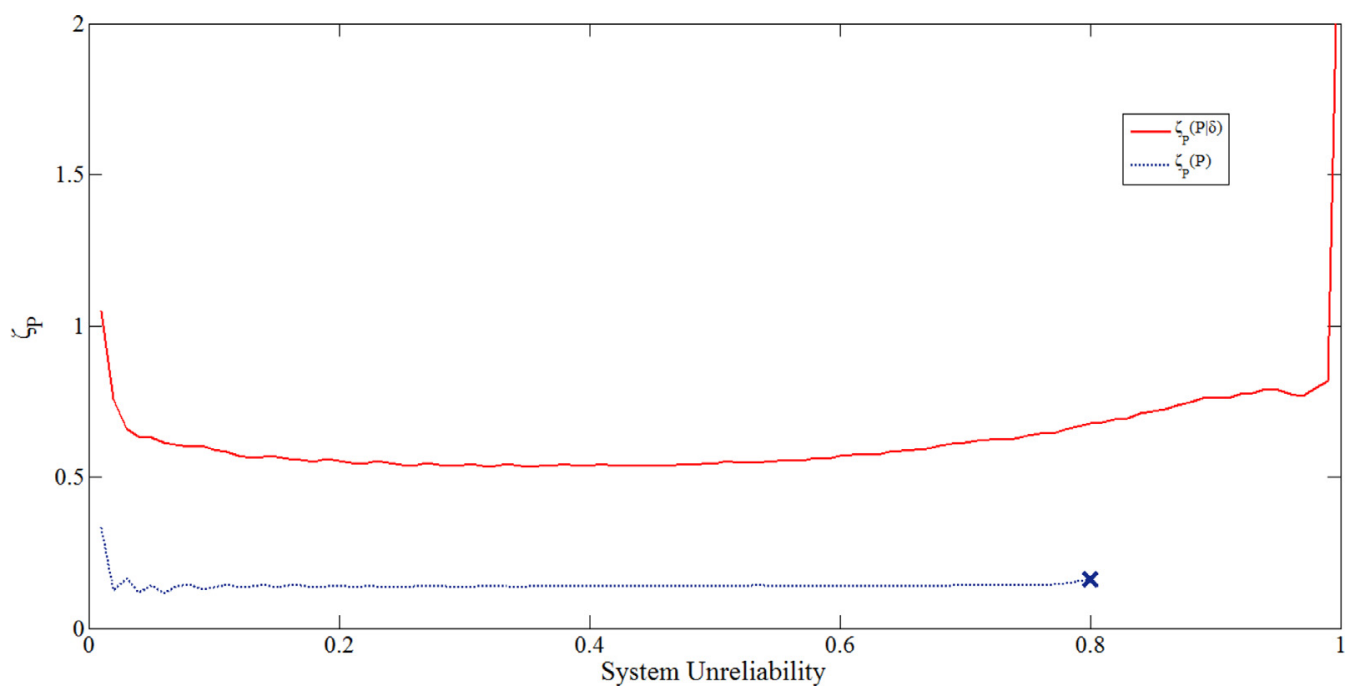

Fig. 20. Relative age interval width.

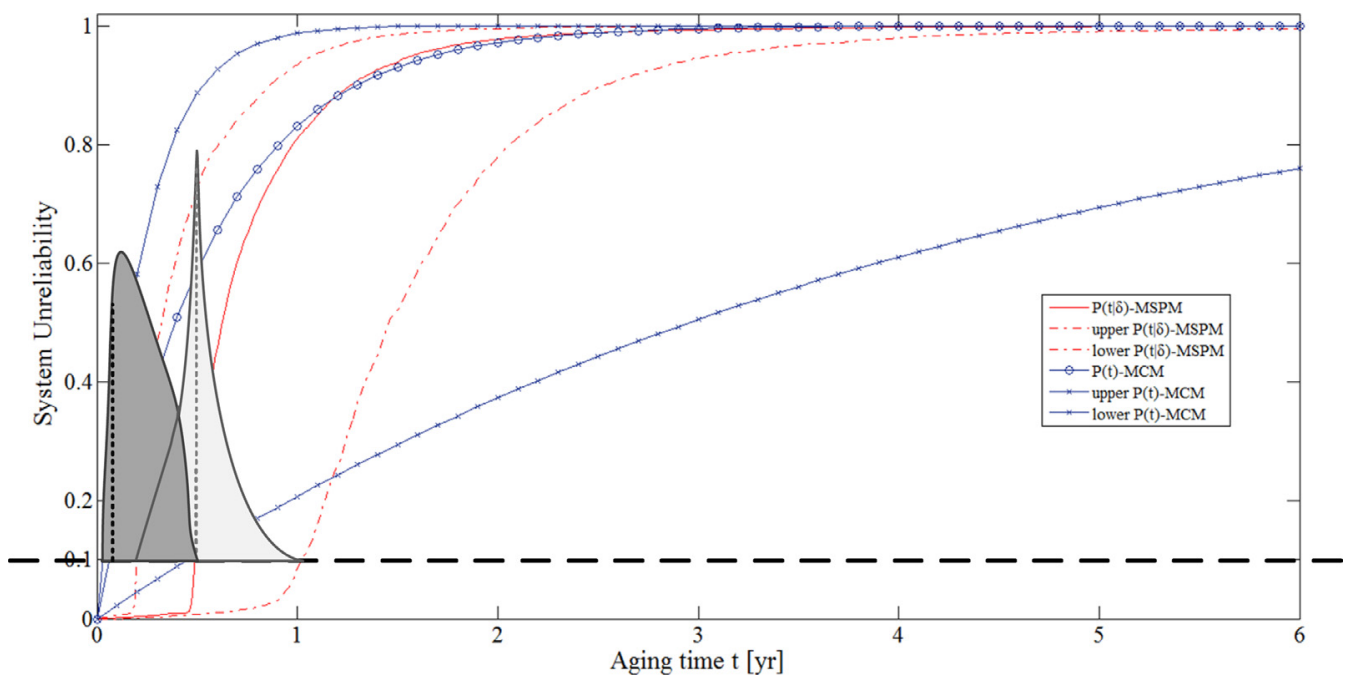

Fig. 21. Example of empirical distributions of the failure times at $P=0.1$. 


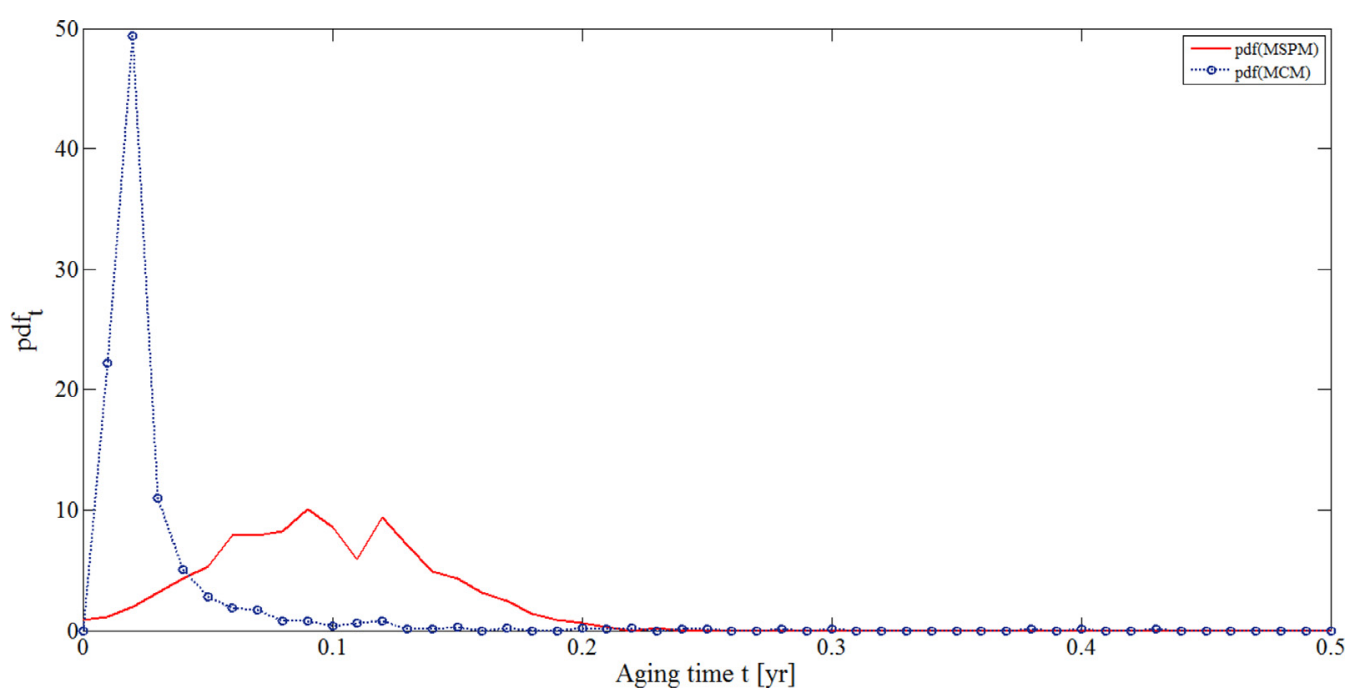

Fig. 22. pdf of the MCM estimates vs. pdf of the MSPM estimates at $P=0.1$.

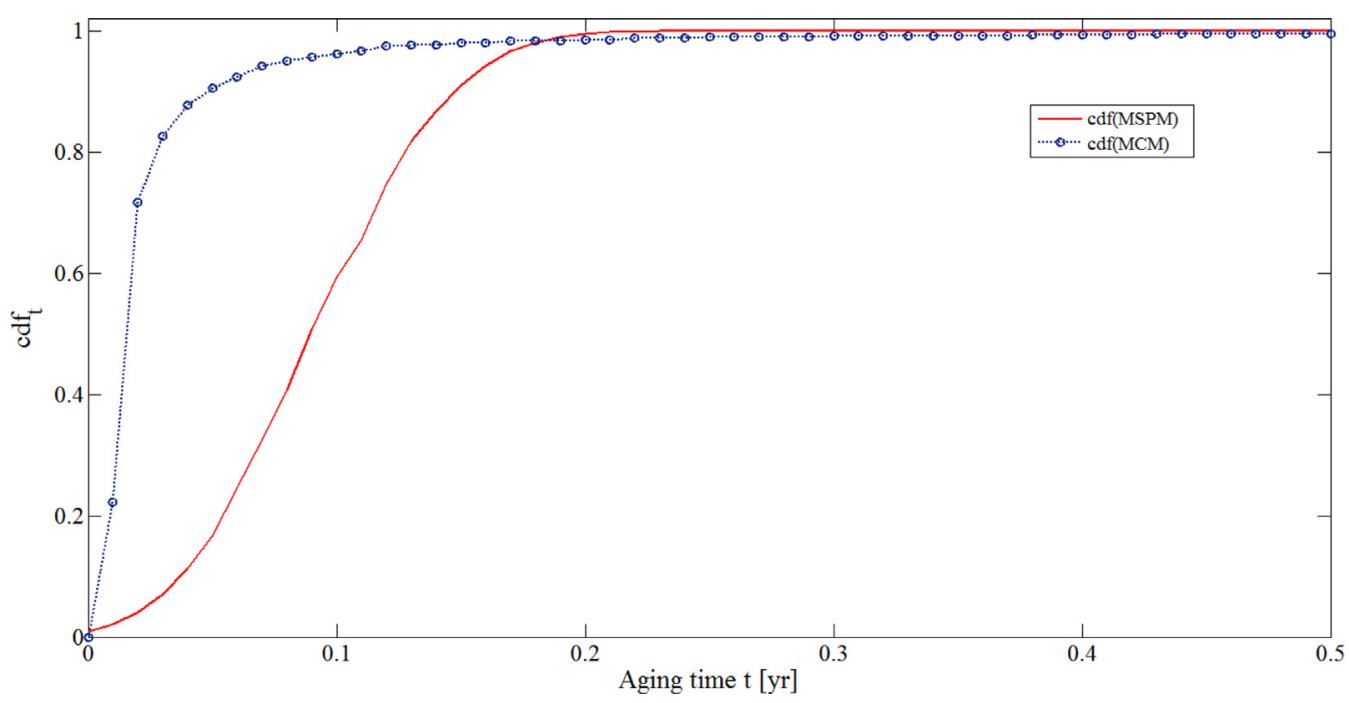

Fig. 23. cdf of the MCM estimates vs. cdf of the MSPM estimates at $P=0.1$.

loop MC simulation for the RPS-MSPM of Section 3, Figs. 22 and 23 are built with the pdf and cdf curves of the system failure times at $P=0.1$, respectively. The pdf of the MCM, as well as its cdf, skew towards the earlier values, compared with the pdf of the MSPM, revealing the more possible early-estimation of the failure times, if the decision maker resorts to a MCM.

\section{Conclusions}

In this paper, a three-loop MC simulation is proposed to properly quantify the effect of uncertain aging- and environmental-dependent transition rates of a MSPM for system reliability assessment. The demanding knowledge and information requirements to build a system MSPM calls for the identification of the components most affecting the system reliability in order to limit the modeling efforts. The obtained component-level MSPM is, then, embedded into a system-level accurate model that can guarantee less uncertainty on the system unreliability estimation, compared to a binary-state modeling approach such as the MCM.

The application of the three-loop MC approach to a RPS system shows the twofold potential benefits of integrating the RTD physics knowledge into the system reliability modeling: on one hand, the narrower confidence interval of the system unreliability of the RPS-MSPM with respect to the RPS-MCM would more likely induce the decisionmaker to rely on the reliability assessment measures provided by the MSPM, whereas, on the other hand, the approach allows balancing modeling efforts and computational demand with accuracy of the results.

\section{References}

[1] Al-Dahidi S, Di Maio F, Baraldi P, Zio E. Remaining useful life estimation in heterogeneous fleets working under variable operating conditions. Reliability Eng Syst Saf 2016;156:109-24.

[2] Aven T, Zio E. Some considerations on the treatment of uncertainties in risk assessment for practical decision making. Reliability Eng Syst Saf 2011;96(1):64-74.

[3] Aven T, Baraldi P, Flage R, Zio E. Uncertainty in risk assessment: the representation and treatment of uncertainties by probabilistic and non-probabilistic methods. John Wiley \& Sons; 2014.

[4] Balaban E, Saxena A, Bansal P, Goebel KF, Curran S. Modeling, detection, and disambiguation of sensor faults for aerospace applications. Sens J, IEEE 2009;9(12):1907-17.

[5] Baraldi P, Podofillini L, Mkrtchyan L, Zio E, Dang VN. Comparing the treatment of uncertainty in bayesian networks and fuzzy expert systems used for a human reliability analysis application. Reliability Eng Syst Saf 2015;138:176-93.

[6] Baraldi P, Di Maio F, Genini D, Zio E. Comparison of data-driven reconstruction methods for fault detection. IEEE Trans Reliability 2015;64(3):852-60.

[7] Borgonovo E. Measuring uncertainty importance: investigation and comparison of alternative approaches. Risk Anal 2006;26(5):1349-61.

[8] Borgonovo E, Castaings W, Tarantola S. Model emulation and moment-independent sensitivity analysis: an application to environmental modelling. Environ Model Softw 2012;34:105-15. 
[9] Borgonovo E, Hazen GB, Plischke E. A common rationale for global sensitivity measures and their estimation. Risk Anal 2016;36(10):1871-95.

[10] Cadini F, Zio E, Di Maio F, Kopustinskas V, Urbonas R. A neural-network-based variance decomposition sensitivity analysis. Int J Nucl Knowl Manage 2007;2(3):299-312.

[11] Carlos S, Sánchez A, Ginestar D, Martorell S. Using finite mixture models in thermal-hydraulics system code uncertainty analysis. Nucl Eng Des 2013;262:306-18.

[12] Catelani M, Ciani L, Venzi M. Sensitivity analysis with MC simulation for the failure rate evaluation and reliability assessment. Measurement 2015;74:150-8.

[13] Cui L, Lü Z, Zhao X. Moment-independent importance measure of basic random variable and its probability density evolution solution. Sci China Technol Sci 2010;53(4):1138-45.

[14] Diaconis Persi, Zabell SandyL. Updating subjective probability. J Am Stat Assoc 1982;77(380):822-30.

[15] Di Maio F, Compare M, Mattafirri S, Zio E. A double-loop Monte Carlo approach for part life database reconstruction and scheduled maintenance improvement. In: European safety and reliability conference, ESREL 2014; 2014. p. 1877-84.

[16] Di Maio F, Nicola G, Zio E, Yu Y. Ensemble-based sensitivity analysis of a best estimate thermal hydraulics model: application to a passive containment cooling system of an AP1000 Nuclear Power Plant. Ann Nucl Energy 2014;73:200-10.

[17] Di Maio F, Colli D, Zio E, Liu T, Tong JJ. A Multi-State Physics Modeling approach for the reliability assessment of Nuclear Power Plants piping systems. Ann Nucl Energy 2015;80:151-65.

[18] Dubois D. Representation, propagation, and decision issues in risk analysis under incomplete probabilistic information. Risk anal 2010;30(3):361-8.

[19] Ericson CA. Hazard analysis techniques for system safety. Hobokrn, New Jersey: John Wiley \& Sons, Inc; 2005.

[20] Gibbs AlisonL, Edward Su Francis. On choosing and bounding probability metrics. Int Stat Rev 2002;70(3):419-35.

[21] Garg H. Performance analysis of complex repairable industrial systems using PSO and fuzzy confidence interval based methodology. ISA Trans 2013;52(2):171-83.

[22] Garg H. Analyzing the behavior of an industrial system using fuzzy confidence interval based methodology. National Acad Sci Lett 2014;37(4):359-70.

[23] Hashemian HM. Measurement of dynamic temperatures and pressures in Nuclear Power Plants Ph.D. Thesis; 2011.

[24] Hoseyni SM, Di Maio F, Vagnoli M, Zio E, Pourgol-Mohammad M. A Bayesian ensemble of sensitivity measures for severe accident modeling. Nucl Eng Des 2015;295:182-91.

[25] IAEA, 1992. Case study on the use of PSA methods: assessment of technical specifications for the Reactor Protection System instrumentation. IAEA-TECDOC-669.

[26] Kendall M, Stuart A. The advanced theory of statistics. Distribution theory, Vol. 1; 1977. London: Griffin.

[27] Kullback S, Leibler RA. On information and sufficiency. Ann Math Stat 1951;22:7986. http://dx.doi.org/10.1214/aoms/1177729694.

[28] Kumar K, Krishna H, Garg R. Estimation of $\mathrm{P}(\mathrm{Y}<\mathrm{X})$ in lindley distribution using progressively first failure censoring. Int J Syst Assur Eng Manage 2015;6(3):330-41.

[29] Li YF, Zio E, Lin YH. A Multi-State Physics Model of component degradation based on stochastic Petri nets and simulation. IEEE Trans Reliability 2012;61(4):921-31.

[30] Lin YH, Li YF, Zio E. Integrating random shocks into Multi-State Physics Models of degradation processes for component reliability assessment. Reliability, IEEE Trans Reliability 2015;64(1):154-66.
[31] McKay, M.D., 1996. "Variance-based methods for assessing uncertainty importance in NUREG-1150 analyses", LA-UR-96-2695, Los Alamos National Laboratory, pp. 7-27.

[32] Norris JR. Markov chains (No. 2008). Cambridge University Press; 1998.

[33] Rocco CM, Zio E. Global sensitivity analysis in a Multi-State Physics Model of component degradation based on a hybrid state-space enrichment and polynomial chaos expansion approach. IEEE Trans Reliability 2013;62(4):781-8.

[34] Saltelli A, Chan K, Scott EM. Sensitivity analysis (Vol. 1). New York: Wiley; 2000.

[35] Saltelli A, Ratto M, Andres T, Campolongo F, Cariboni J, Gatelli D, et al. Global sensitivity analysis: the primer. Chichester: John Wiley \& Sons Ltd; 2008.

[36] Sobol IM. Global sensitivity indices for nonlinear mathematical models and their Monte Carlo estimates. Math Comput Simulation 2001;55(1):271-80.

[37] Swanson C. Optimal temperature sensor selection: achieving accurate temperature measurement. Euro-Asia Semiconductor 2007;29(7):23-8.

[38] Unwin SD, Lowry PP, Layton RF, Heasler PG, Toloczko MB. Multi-state physics models of aging passive components in probabilistic risk assessment (No. PNNL-SA-76893). Richland, WA (US): Pacific Northwest National Laboratory (PNNL); 2011.

[39] US: EPRI, 2008. "Advanced Light Water Reactor Utility Requirements Document, (Annex A reliability data base for passive ALWR PRAs)".

[40] Wang W, Di Maio F, Zio E. Component- and system-level degradation modeling of digital instrumentation and control systems based on a Multi-State Physics Modeling approach. Ann Nucl Energy 2016;95:135-47.

[41] Wang W, Zhao J, Tong JJ, Zhou JX, Xiao P. Evaluation method of reliability indicator of Reactor Protection System. Atomic Energy Sci Technol 2015;49(6):1101-8.

[42] Wei P, Lu Z, Yuan X. Monte Carlo simulation for moment-independent sensitivity analysis. Reliability Eng Syst Saf 2013;110:60-7.

[43] Yu W, Harris TJ. Parameter uncertainty effects on variance-based sensitivity analysis. Reliability Eng Syst Saf 2009;94(2):596-603.

[44] Yu Y, Liu T, Tong J, Zhao J, Di Maio F, Zio E, et al. Multi-experts ana-lytic hierarchy process for the sensitivity analysis of passive safety systems. In: Proceedings of the 10th international probabilistic safety assessment \& management conference; 2010 PSAM10, Seattle, June 2010.

[45] Yun D, Yacout AM, Vilim RB. Modeling the aging effects of Nuclear Power Plant resistance temperature detectors. 8th international topical meeting on nuclear plant instrumentation, control, and human-machine interface technologies 2012, NPIC and HMIT 2012: enabling the future of nuclear energy; 2012.

[46] Zhang R, Mahadevan S. Integration of computation and testing for reliability estimation. Reliability Eng Syst Saf 2001;74(1):13-21.

[47] Zhang H, Mullen RL, Muhanna RL. Interval Monte Carlo methods for structural reliability. Struct Saf 2010;32(3):183-90.

[48] Zhang Y, Liu Y, Yang X. Parametric sensitivity analysis for importance measure on failure probability and its efficient Kriging solution. Math Probl Eng 2015;2015 Article ID 685826, 13 pages. doi:10.1155/2015/685826.

[49] Zio E. An introduction to the basics of reliability and risk analysis. World Sci 2007; 13:147-52.

[50] Zio E, Pedroni N. Building confidence in the reliability assessment of thermal-hydraulic passive systems. Reliability Eng Syst Saf 2009;94(2):268-81.

[51] Zio E, Pedroni N. Monte Carlo simulation-based sensitivity analysis of the model of a thermal-hydraulic passive system. Reliability Eng Syst Saf 2012;107:90-106.

[52] Zio E. The Monte Carlo simulation method for system reliability and risk analysis. London: Springer; 2013. 